Geometry ${ }^{6} \mathcal{T}$ Topology

Volume 3 (1999) 269-302

Published: 11 September 1999

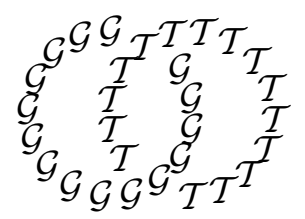

\title{
Non-positively curved aspects of Artin groups of finite type
}

\author{
Mladen Bestvina \\ Department of Mathematics, University of Utah \\ Salt Lake City, UT 84112, USA \\ Email: bestvina@math.utah.edu
}

\begin{abstract}
Artin groups of finite type are not as well understood as braid groups. This is due to the additional geometric properties of braid groups coming from their close connection to mapping class groups. For each Artin group of finite type, we construct a space (simplicial complex) analogous to Teichmüller space that satisfies a weak nonpositive curvature condition and also a space "at infinity" analogous to the space of projective measured laminations. Using these constructs, we deduce several group-theoretic properties of Artin groups of finite type that are well-known in the case of braid groups.
\end{abstract}

AMS Classification numbers Primary: 20F32, 20F36

Secondary: 55P20

Keywords: Artin groups, nonpositive curvature

Proposed: Walter Neumann

Seconded: Wolfgang Metzler, Joan Birman
Received: 27 November 1998

Revised: 5 August 1999

Copyright Geometry and Topology 


\section{Introduction and review}

A theme in geometric group theory has been to, on one hand, construct spaces with rich geometry where (given) interesting groups act by isometries, and on the other hand, show that such actions have useful group-theoretic consequences. A particularly successful sort of a geometric structure one considers is a CAT(0) metric. Experience shows that the class of groups that act cocompactly and isometrically on $\mathrm{CAT}(0)$ spaces is large and includes many standard groups, while at the same time the groups in this class have a rich structure (see [3]). For example, the following properties hold in this class:

- There are only finitely many conjugacy classes of finite subgroups.

- Every solvable subgroup is finitely generated and virtually abelian.

- The set of translation lengths of elements of infinite order is bounded away from 0 .

In this paper, we focus on the class of Artin groups of finite type (and their quotients by the center). We review the basic definitions and results in the next two sections. Tom Brady [7] has shown that Artin groups of finite type with $\leq 3$ generators belong to the $\mathrm{CAT}(0)$ class and he has proposed a piecewise Euclidean complex with a metric for each Artin group of finite type. Daan Krammer has checked that this complex is $\operatorname{CAT}(0)$ for the braid group on 5 strands.

It is a result of Ruth Charney [12] that Artin groups of finite type are biautomatic. This fact has a number of group-theoretic consequences; in particular, by a result of Gersten-Short [17], the translation length of each element of infinite order is positive, and consequently every nilpotent subgroup is virtually abelian.

In this paper, we will construct a contractible simplicial complex on which a given Artin group of finite type acts, and show that there is a natural "metric" on it, preserved by the group action, that satisfies a property somewhat weaker than CAT(0). The word "metric" is in quotes because it is not symmetric. This structure is, however, sufficient to deduce the same group-theoretic properties as in the case of $\operatorname{CAT}(0)$ metrics.

The main tool in this paper is the left greedy normal form for the elements of Artin groups of finite type. The basic properties of this normal form were established in the inspiring paper of Deligne [15], and also in Brieskorn-Saito [9], both of which build on the work of Garside [16]. Charney [12] showed 
that this form gives rise to a biautomatic structure. Deligne also showed that the quotient by the Coxeter group of associated hyperplane complement is an Eilenberg-Mac Lane space for each Artin group of finite type. Since these manifolds can easily be compactified, Artin groups of finite type have finite Eilenberg-Mac Lane spaces.

Aside from establishing the above group-theoretic properties of Artin groups of finite type and their central quotients, we will give a simple proof of Squier's theorem [18] that these groups are duality groups. Moreover, we show that they are highly connected at infinity. (One way to show that a group is a duality group of dimension $d$ is to argue that the group is $d$-dimensional and $(d-2)-$ connected at infinity.) We also show that every normal abelian subgroup is central. This answers a question of Jim Carlson that provided the impetus to study the geometry of Artin groups of finite type.

We summarize the geometric features of the complex in the following (see the next section for definitions of $S$ and $\Delta$ ):

Main Theorem Let $\mathcal{A}$ be an Artin group of finite type and $\mathcal{G}=\mathcal{A} / \Delta^{2}$ the quotient of $\mathcal{A}$ by the central element $\Delta^{2}$. Then $\mathcal{G}$ acts simplicially on a simplicial complex $X=X(\mathcal{G})$ with the following properties:

- The action is cocompact and transitive on the vertices (section 2.1).

- $X$ is a flag complex (section 2.1).

- $X$ is contractible, $(\operatorname{card}(S)-3)$-connected at infinity and proper homotopy equivalent to a $(\operatorname{card}(S)-1)$-complex (Theorem 3.6).

- Each pair of vertices of $X$ is joined by a preferred edge-path, called a geodesic (section 3.1). The collection of geodesics is invariant under the following operations: translation by group elements, subpaths, inverses (Proposition 3.1), and concatenations with nontrivial overlap.

- There is a function $d=d_{w d}: X^{(0)} \times X^{(0)} \rightarrow\{0,1,2, \cdots\}$ (a "nonsymmetric distance function") satisfying the triangle inequality and

$$
d(v, w)=0 \Longleftrightarrow v=w
$$

(Proposition 3.1). Further, $d_{w d}$ is comparable to the edge-path metric in $X$ (Lemma 3.3).

- If $v_{0}, v_{1}, v_{2}, \cdots v_{n}$ are the consecutive vertices along a geodesic, then

$$
d\left(v_{0}, v_{n}\right)=\sum_{i=1}^{n} d\left(v_{i-1}, v_{i}\right)
$$


- If the associated Coxeter group $\mathcal{W}$ is irreducible and nonabelian, then every geodesic can be extended in both directions to a longer geodesic (Proposition 4.9).

- (Theorem 3.13) For any three vertices $a, b, c \in X$ and any vertex $p \neq b, c$ on the geodesic from $b$ to $c$ we have

$$
d(a, p)<\max \{d(a, b), d(a, c)\} .
$$

- The set of vertices of each simplex in $X$ has a canonical cyclic ordering which is invariant under the group action and is compatible with the passage to a face (Lemma 4.6).

The dimension of the complex we construct is higher than the expected dimension (ie, the dimension of the group). It is, however, often the case that one has to increase the dimension in order to obtain better geometric properties, $\mathrm{cf}$ non-uniform lattices, Teichmüller space, Outer Space.

Perhaps the most interesting remaining unresolved group-theoretic questions about Artin groups $\mathcal{A}$ of finite type are the following:

Question 1 Does $\mathcal{A}$ satisfy the Tits Alternative, ie, if $H<\mathcal{A}$ is a subgroup which is not virtually abelian, does $H$ necessarily contain a nonabelian free group?

Question 2 Is $\mathcal{A}$ virtually poly-free?

The Tits Alternative is not known for CAT(0) groups and it seems unlikely that the techniques of this paper will resolve Question 1. A group $G$ is poly-free if there is a finite sequence

$$
G=G_{0} \supset G_{1} \supset G_{2} \supset \cdots \supset G_{n}=\{1\}
$$

such that each $G_{i}$ is normal in $G_{i-1}$ and the quotient $G_{i-1} / G_{i}$ is free. It would seem reasonable to expect that the pure Artin group $P \mathcal{A}$ (the kernel of the homomorphism to the associated Coxeter group) is poly-free, with the length of the series above equal to the dimension (ie, the number of generators), and all successive quotients of finite rank. This was verified by Brieskorn [8] for types $A_{n}$ (this is classical), $B_{n}, D_{n}, I_{2}(p)$, and $F_{4}$. Of course, the positive answer to Question 2 implies the positive answer to Question 1. Another approach to Question 1 would be to find a faithful linear representation of each Artin group of finite type. This seems rather difficult (if not impossible) even for braid groups.

Questions 1 and 2 can be asked in the setting of Artin groups of infinite type as well. However, more basic questions are still open in that setting; the most 
striking is whether all (finitely generated) Artin groups admit a finite $K(\pi, 1)$. Recently, Charney and Davis [11],[10] have made substantial progress toward this question, but the general case remains open.

We end the paper by constructing a space at infinity analogous to the space of projectivized measured geodesic laminations in the case of mapping class groups.

Acknowledgements I benefited from discussions about Artin groups with several people. I would like to thank John Luecke and Alan Reid for having the patience to listen to me while I was learning about Artin groups. Discussions with Warren Dicks have shed much light on the difficulties in Question 2. I would also like to thank the referee for suggesting simplifications of some of the arguments.

Support by the National Science Foundation is gratefully acknowledged.

\section{$1.1 \quad$ Coxeter groups}

We follow the notation of [10]. For proofs of the facts listed below see [6]. Let $S$ be a finite set. A Coxeter matrix is a symmetric function $m: S \times S \rightarrow$ $\{1,2,3, \cdots, \infty\}$ such that $m(s, s)=1$ for all $s \in S$ and $m\left(s, s^{\prime}\right) \geq 2$ for $s \neq s^{\prime}$. The associated Coxeter group is the group $\mathcal{W}$ given by the presentation

$$
\mathcal{W}=\left\langle S \mid\left(s s^{\prime}\right)^{m\left(s, s^{\prime}\right)}=1\right\rangle
$$

where $m=\infty$ means no relation. If $S^{\prime}$ is a proper subset of $S$ and $m^{\prime}$ is the restriction of $m$ to $S^{\prime} \times S^{\prime}$, then there is a natural homomorphism

$$
\mathcal{W}^{\prime}=\left\langle S^{\prime} \mid\left(s s^{\prime}\right)^{m^{\prime}\left(s, s^{\prime}\right)}=1\right\rangle \longrightarrow \mathcal{W}
$$

and this homomorphism is injective. The images of such homomorphisms are called special (Coxeter) subgroups of $\mathcal{W}$.

In this paper we will consider only finite Coxeter groups $\mathcal{W}$, and this is what we assume from now on.

There is a canonical faithful orthogonal representation of $\mathcal{W}$ on a finite-dimensional vector space $V=V_{\mathcal{W}}$ (of dimension $\left.\operatorname{dim} V=\operatorname{card}(S)\right)$ such that:

- Each generator $s \in S$ acts as a reflection, ie, it fixes a codimension 1 subspace of $V$. These subspaces and their $\mathcal{W}$-translates are called walls.

- The closures in $V$ of the complementary components of the union of the walls are the chambers. They are simplicial cones, and $\mathcal{W}$ acts on the set of chambers simply transitively. 
- Special subgroups and their conjugates are precisely the stabilizers of nonzero points in $V$.

- There is a chamber $Q$, called the fundamental chamber, that intersects the fixed sets of each $s \in S$ in top-dimensional faces. When $\mathcal{W}$ is irreducible, $Q$ and $-Q$ are the only such chambers.

- The longest element is the unique element $\Delta \in \mathcal{W}$ that takes $Q$ to $-Q$ (and vice versa). It has order 2 , and conjugation by $\Delta$ induces an involution of $S$.

Two chambers $Q^{\prime}$ and $Q^{\prime \prime}$ are adjacent if $Q^{\prime} \cap Q^{\prime \prime}$ is contained in a wall and has nonempty interior in the wall. This wall is then the unique wall that separates $Q^{\prime}$ from $Q^{\prime \prime}$, and we say that it abuts $Q^{\prime}$ and $Q^{\prime \prime}$. A sequence $Q_{0}, Q_{1}, \cdots, Q_{n}$ of chambers is a gallery $G$ of length $n$ (from $Q_{0}$ to $Q_{n}$ ) if $Q_{i}$ and $Q_{i+1}$ are adjacent for $i=0,1,2, \cdots, n-1$. Any path in $U$ which is transverse to the collection of the walls determines a gallery and every gallery arises in this way. Any two chambers are connected by a gallery. The distance between two chambers $A, B$ is the length of a shortest gallery connecting them; equivalently, it is the number of walls separating $A$ and $B$. A gallery is geodesic if its length is equal to the distance between the initial and the terminal chamber. Equivalently, a gallery is geodesic if it crosses each wall at most once (we say that a gallery crosses a wall if any associated path does, ie, if the wall separates a pair of consecutive chambers). For example, straight line segments transverse to the collection of walls determine geodesic galleries.

A Coxeter group $\mathcal{W}$ is irreducible if there is no nontrivial partition $S=S_{1} \sqcup S_{2}$ with $m\left(s_{1}, s_{2}\right)=2$ whenever $s_{1} \in S_{1}$ and $s_{2} \in S_{2}$.

\subsection{Artin groups}

If $S$ is a finite set and $m: S \times S \rightarrow\{2,3, \cdots, \infty\}$ a Coxeter matrix, the associated Artin group is defined to be the group

$$
\mathcal{A}=\left\langle S \mid \operatorname{prod}\left(s, s^{\prime}, m\left(s, s^{\prime}\right)\right)=\operatorname{prod}\left(s^{\prime}, s, m\left(s^{\prime}, s\right)\right)\right\rangle
$$

where

$$
\operatorname{prod}\left(s, s^{\prime}, m\right)=\underbrace{s s^{\prime} s s^{\prime} \cdots}_{m \text { times }}
$$

and again $m=\infty$ means no relation.

There is a natural homomorphism $\pi: \mathcal{A} \rightarrow \mathcal{W}, \pi(s)=s$. The kernel is the pure Artin group $\mathcal{P}$. If $S^{\prime}$ is a subset of $S$, let $\mathcal{A}^{\prime}$ be the Artin group associated 
with the restriction of $m$ to $S^{\prime} \times S^{\prime}$. We then have a natural homomorphism $\mathcal{A}^{\prime} \rightarrow \mathcal{A}, s^{\prime} \mapsto s^{\prime}$. This homomorphism is injective.

By $\mathcal{A}^{+}$we denote the submonoid of $\mathcal{A}$ generated by $S$, ie, the monoid of positive words. As shown by Deligne and Brieskorn-Saito, if two positive words represent the same element of $\mathcal{A}$, then they can be transformed to each other by repeated substitutions given by the defining relations. A positive word $s_{i_{1}} s_{i_{2}} \cdots s_{i_{k}}$ can be geometrically thought of as a gallery of length $k$ starting at $Q: Q, s_{i_{1}}(Q), s_{i_{1}} s_{i_{2}}(Q), \cdots, s_{i_{1}} s_{i_{2}} \cdots s_{i_{k}}(Q)$. We will also consider any $\mathcal{W}$-translate of this gallery as being associated with the same positive word. Multiplication in $\mathcal{A}^{+}$corresponds to the concatenation of galleries (after a possible $\mathcal{W}$-translation so that the last chamber of the first gallery coincides with the first chamber of the second gallery).

\subsection{Normal form in $\mathcal{A}^{+}$and $\mathcal{A}$}

Following Deligne, for $x, y \in \mathcal{A}^{+}$we write $x<y$ if there is $z \in \mathcal{A}^{+}$such that $y=x z$. We say that $A \in \mathcal{A}^{+}$is an atom if its word-length in $\mathcal{A}^{+}$is equal to the word-length of $\pi(A) \in \mathcal{W}$, or equivalently if a gallery associated to $A$ is geodesic. The homomorphism $\pi: \mathcal{A} \rightarrow \mathcal{W}$ induces a bijection between the set of atoms and $\mathcal{W}$. We will often identify an atom $A$ with the associated chamber $\pi(A)(Q)$. The atom that corresponds to the longest element $\Delta$ is also denoted $\Delta$. We have $1<A<\Delta$ for all atoms $A . \Delta^{2}$ is central in $\mathcal{A}$ and conjugation by $\Delta$ induces an involution of $\mathcal{A}$ that extends the involution $S \rightarrow S$ mentioned above. This involution will be denoted by $x \mapsto \bar{x}$. It restricts to involutions of $\mathcal{A}^{+}$and of the set of atoms.

The following two propositions are proved in Deligne's paper [15].

Proposition 1.1 Suppose that $\Omega$ is a nonempty finite subset of $\mathcal{A}^{+}$such that

(1) $x \in \Omega, x^{\prime}<x$ implies $x^{\prime} \in \Omega$,

(2) if $\sigma, \tau \in S$ are two generators, $x \in \mathcal{A}^{+}, x \sigma, x \tau \in \Omega$, then

$$
\operatorname{xprod}(\sigma, \tau, m(\sigma, \tau)) \in \Omega .
$$

Then there is a unique $y \in \mathcal{A}^{+}$such that

$$
\Omega=\left\{x \in \mathcal{A}^{+} \mid x<y\right\} .
$$

Further, for any $y$ the set $\Omega=\left\{x \in \mathcal{A}^{+} \mid x<y\right\}$ satisfies 1 and 2 . 
In particular, for $x_{1}, x_{2} \in \mathcal{A}^{+}$we can define $x_{1} \wedge x_{2} \in \mathcal{A}^{+}$as the largest element $z \in \mathcal{A}^{+}$such that $z<x_{1}$ and $z<x_{2}$. The existence of the largest such element follows by applying Proposition 1.1 to the set $\Omega=\left\{z \mid z<x_{1}, z<x_{2}\right\}$.

There is a function reverse: $\mathcal{A}^{+} \rightarrow \mathcal{A}^{+}$given by reverse $\left(s_{i_{1}} s_{i_{2}} \cdots s_{i_{k}}\right)=$ $s_{i_{k}} \cdots s_{i_{2}} s_{i_{1}}$. If $x \in \mathcal{A}^{+}$is represented by a gallery $Q_{0}, Q_{1}, \cdots, Q_{k-1}, Q_{k}$, then $\operatorname{reverse}(x)$ is represented by the gallery $Q_{k}, Q_{k-1}, \cdots, Q_{1}, Q_{0}$.

For every atom $A$ there is an atom $A^{*}$ such that $A A^{*}=\Delta$ (and there is also an atom ${ }^{*} A$ such that ${ }^{*} A A=\Delta$ ). Every $x \in \mathcal{A}^{+}$can be represented as the product of atoms (or even generators), say $x=A_{1} A_{2} \cdots A_{k}$. Then we have

$$
x A_{k}^{*} \bar{A}_{k-1}^{*} A_{k-2}^{*} \cdots=\Delta^{k}
$$

so that $x<\Delta^{k}$. Further, if $x, y<\Delta^{k}$, and if $\tilde{x}, \tilde{y} \in \mathcal{A}^{+}$are such that $x \tilde{x}=\Delta^{k}$ and $y \tilde{y}=\Delta^{k}$, then $x<y \Longleftrightarrow \operatorname{reverse}(\tilde{y})<\operatorname{reverse}(\tilde{x})$ and we deduce (from the existence of $\tilde{x} \wedge \tilde{y}$ ) that for any $x, y \in \mathcal{A}^{+}$there is a unique $x \vee y \in \mathcal{A}^{+}$ such that

- $x<x \vee y$,

- $y<x \vee y$, and

- $x<z, y<z$ implies $x \vee y<z$.

For every $x \in \mathcal{A}$ there is $k \geq 0$ such that $\Delta^{k} x \in \mathcal{A}^{+}$. This reduces the understanding of $\mathcal{A}$ to the understanding of $\mathcal{A}^{+}$. There is no such reduction when the associated Coxeter group is infinite.

Proposition 1.2 For every $g \in \mathcal{A}^{+}$there is a unique atom $\alpha(g)$ such that

(1) $\alpha(g)<g$, and

(2) if $A$ is an atom with $A<g$, then $A<\alpha(g)$.

Furthermore, $\alpha(x y)=\alpha(x \alpha(y))$ for all $x, y \in \mathcal{A}^{+}$.

The left greedy normal form of $g \in \mathcal{A}^{+}$is the representation of $g$ as the product of nontrivial atoms

$$
g=A_{1} A_{2} \cdots A_{k}
$$

such that $A_{i}=\alpha\left(A_{i} A_{i+1} \cdots A_{k}\right)$ for all $1 \leq i \leq k$. To emphasize that the above product of atoms is the normal form of $g$, we will write $g=A_{1} \cdot A_{2} \cdots A_{k}$. Clearly, the normal form for $g$ is unique. A product $A_{1} A_{2} \cdots A_{k}$ is a normal form iff each subproduct of the form $A_{i} A_{i+1}$ is a normal form. If $A_{1} \cdot A_{2} \cdots \cdots A_{k}$ is a normal form, then so is $\bar{A}_{1} \cdot \bar{A}_{2} \cdots \bar{A}_{k}$. 
$\Delta$ 's appear at the beginning of the normal form, ie, if $A_{1} \cdot A_{2} \cdots A_{k}$ is a normal form, then there is $j$ such that $A_{1}=A_{2}=\cdots=A_{j}=\Delta$ and $A_{i} \neq \Delta$ for $i>j$.

Geometric interpretation of the normal form is given by the following result.

Proposition 1.3 Let $A$ and $B$ be nontrivial atoms, with $A$ represented by a geodesic gallery from $Q_{1}$ to $Q_{2}$ and $B$ by a geodesic gallery from $Q_{2}$ to $Q_{3}$. Then $A \cdot B$ is a normal form iff no wall that abuts $Q_{2}$ has $Q_{1}$ and $Q_{2}$ on one side and $Q_{3}$ on the other side.

There is the homomorphism length: $\mathcal{A} \rightarrow \mathbb{Z}$ that sends each $s \in S$ to $1 \in \mathbb{Z}$. This homomorphism restricts to the word-length on $\mathcal{A}^{+}$.

We will need a straightforward generalization of Proposition 1.2.

Proposition 1.4 Suppose $x \in \mathcal{A}^{+}$and $y_{1} \cdot y_{2} \cdots y_{l}$ is a normal form in $\mathcal{A}^{+}$. Suppose the product $x\left(y_{1} \cdot y_{2} \cdots y_{l}\right)$ has normal form

$$
z_{1} \cdot z_{2} \cdots z_{m}
$$

Then for each $i \leq l$ the normal form of the product $x\left(y_{1} y_{2} \cdots y_{i}\right)$ begins with

$$
z_{1} \cdot z_{2} \cdots z_{i} \cdots
$$

Proof For $i=1$ this follows from Proposition 1.2. We argue by induction on $i$. It suffices to show that $z_{1} z_{2} \cdots z_{i}<x y_{1} y_{2} \cdots y_{i}$. By Proposition 1.2 we have $z_{1}<x y_{1}$ so we can write $x y_{1}=z_{1} w$ for some $w \in \mathcal{A}^{+}$. Then $x y_{1} y_{2} \cdots y_{l}=z_{1} z_{2} \cdots z_{m}$, after cancelling on the left, implies that $w y_{2} \cdots y_{l}=$ $z_{2} \cdots z_{m}$ and the right-hand side is visibly a normal form. By induction it follows that $z_{2} \cdots z_{i}<w y_{2} \cdots y_{i}$. Now multiply on the left by $z_{1}$ to obtain $z_{1} z_{2} \cdots z_{i}<x y_{1} y_{2} \cdots y_{i}$.

\section{$2 \mathcal{G}$ and its complex $X(\mathcal{G})$}

\subsection{Definition of $X(\mathcal{G})$}

It is more convenient to study the group $\mathcal{G}=\mathcal{A} / \Delta^{2}$. The groups $\mathcal{G} \times \mathbb{Z}$ and $\mathcal{A}$ are commensurable. Indeed, the homomorphism $\mathcal{A} \rightarrow \mathcal{G} \times \mathbb{Z}$ which is natural projection in the first coordinate and sends each generator to $1 \in \mathbb{Z}$ in the second coordinate (ie, it is the length homomorphism in the second coordinate) 
is a monomorphism onto a finite index subgroup. Moreover, $\mathbb{Z}=\left\langle\Delta^{2}\right\rangle \hookrightarrow \mathcal{A}$ has a splitting with values in $\frac{1}{\text { length }\left(\Delta^{2}\right)} \mathbb{Z}$ (given by length divided by the length of $\left.\Delta^{2}\right)$.

The goal of this section is to describe a contractible simplicial complex $X=$ $X(\mathcal{G})$ on which the group $\mathcal{G}=\mathcal{A} /\left\langle\Delta^{2}\right\rangle$ acts cocompactly and with finite point stabilizers. The vertex set is the coset space

$$
V=\mathcal{A} /\langle\Delta\rangle=\{g\langle\Delta\rangle \mid g \in \mathcal{A}\} .
$$

The group $\mathcal{A}$ acts naturally on the left, and the central element $\Delta^{2}$ acts trivially, so $\mathcal{G}=\mathcal{A} /\left\langle\Delta^{2}\right\rangle$ acts on $V$. The action is transitive, and the stabilizer of the point $\langle\Delta\rangle \in V$ is $\langle\Delta\rangle \cong \mathbb{Z} / 2$.

We call the elements of $V$ the vertices. Each vertex has a representative $g \in \mathcal{A}^{+}$ (obtained by multiplying an arbitrary representative by a high power of $\Delta^{2}$ ). Let $g=\Delta^{m} \cdot B_{1} \cdot B_{2} \cdots B_{k}$ be the normal form for $g$ with $B_{i} \neq \Delta$ for all $i$ (as remarked above, if the normal form of $g$ involves any $\Delta$ 's, they appear at the beginning). Now shift all $\Delta$ 's to the end to obtain a representative in $\mathcal{A}^{+}$ of the same coset whose normal form does not have any $\Delta$ 's. If $m$ is even, this amounts to replacing $g$ by $B_{1} \cdot B_{2} \cdots B_{k}$, and if $m$ is odd $g$ is replaced by $\bar{B}_{1} \cdot \bar{B}_{2} \cdots \bar{B}_{k}$. Such a coset representative is unique, and we will frequently identify $V$ with the set of elements of $\mathcal{A}^{+}$whose normal form has no $\Delta$ 's. The identity element of $\mathcal{A}^{+}$(whose normal form is empty) is viewed as the basepoint, denoted $*$.

The atomnorm of a vertex $v$, denoted $|v|$, is the number of atoms in the normal form of the special representative, ie, the number of atoms not counting $\Delta$ 's in the normal form of any positive representative. Left translation by $\Delta$ sends a special representative $B_{1} \cdot B_{2} \cdots B_{m}$ to a special representative $\bar{B}_{1} \cdot \bar{B}_{2} \cdots \cdots \bar{B}_{m}$ so this involution is atomnorm preserving. It follows that there is a unique left invariant function $d_{a t}: V \times V \rightarrow \mathbb{Z}_{+}=\{0,1,2, \cdots\}$ (ie $d_{a t}(g(v), g(w))=$ $d_{a t}(v, w)$ for all $g \in \mathcal{G}$ and all $\left.v, w \in V\right)$ such that $d_{a t}(*, v)$ is the atomnorm of $v$. We call $d_{a t}$ the atomdistance on $V$.

In order to prove that $d_{a t}$ is symmetric and satisfies the triangle inequality, we need the following lemma.

Lemma 2.1 If $g \in \mathcal{A}^{+}$is the product of $k$ atoms, then the normal form of $g$ has $\leq k$ atoms.

Proof Induction on $k$ starting with $k=1$ when it's clear. For $k=2$ the statement follows from the definition of normal form and the fact that a subgallery 
of a geodesic gallery is a geodesic gallery. Say $g=B_{1} B_{2} \cdots B_{k}$. Inductively, the normal form for $B_{2} \cdots B_{k}$ is $C_{2} \cdot C_{3} \cdots \cdots C_{l}$ with $l \leq k$. If $B_{1} C_{2}$ is an atom, then the normal form for $g$ is $\left(B_{1} C_{2}\right) \cdot C_{3} \cdots \cdots C_{l}$ and has length $l-1<k$. If $B_{1} C_{2}$ is not an atom, then the normal form for $B_{1} C_{2}$ is $X \cdot Y$ (say) and the normal form for $g$ is $X$ followed by the normal form for $Y C_{3} \cdots C_{l}$. The latter has length $<l$ by induction, so the statement is proved.

Proposition $2.2 d_{a t}: V \times V \rightarrow \mathbb{Z}_{+}$is a distance function.

Proof The triangle inequality follows from Lemma 2.1. We argue that $d_{a t}$ is symmetric. By left invariance, it suffices to show that $d_{a t}(*, v)=d_{a t}(v, *)$ for all $v \in V$. Let $g$ be a group element such that $g(v)=*$; we need to argue that the norm of $v$ equals the norm of $g(*)$ (which can be viewed as an "inverse" of $v$; there are two inverses - obtained from each other by applying the "bar" involution). Say $v=B_{1} \cdot B_{2} \cdots B_{k}$ is the normal form (without $\Delta$ 's). Let $C_{i}$ be the unique atom such that $C_{i} B_{i}=\Delta$. Thus $\Delta C_{i} \in \mathcal{G}$ is the inverse of $B_{i}$ and $g$ can be taken to be $\Delta C_{k} \Delta C_{k-1} \cdots \Delta C_{1}$. Thus $g(*)$ is represented by $\bar{C}_{k} C_{k-1} \bar{C}_{k-2} \cdots$, a product of $k$ atoms. By Lemma 2.1, the norm of $g(*)$ is $\leq k$. Applying the same argument with the roles of $v$ and $g(v)$ reversed, we see that the norms of $v$ and $g(v)$ are equal.

Definition 2.3 $X(\mathcal{G})$ is the simplicial complex whose vertex set is $V$ and a collection $v_{0}, v_{1}, \cdots, v_{m} \in V$ spans a simplex iff $d_{a t}\left(v_{i}, v_{j}\right)=1$ for all $i \neq j$.

Thus $X(\mathcal{G})$ is a flag complex, ie, if each pair in a set of vertices bounds an edge in $X(\mathcal{G})$ then this set spans a simplex. The group $\mathcal{G}$ acts on $X(\mathcal{G})$ simplicially and cocompactly. Note that the distance $d_{a t}$ on $V$ is the edge-path distance between the vertices of $X(\mathcal{G})$.

Examples 2.4 We can explicitly see $X(\mathcal{G})$ in simple cases. When $\mathcal{A}=\mathbb{Z} \times \mathbb{Z}$, $X(\mathcal{G})$ is the line. When $\mathcal{A}$ is $\mathbb{Z} \times \mathbb{Z} \times \mathbb{Z}, X$ is the plane (triangulated in the usual $(3,3,3)$-fashion), and more generally, when $\mathcal{A}=\mathbb{Z}^{m}$, then $X(\mathcal{G})$ is Euclidean $(m-1)$-space. When $\mathcal{A}=B_{3}$ is the braid group on 3 strands, $X(\mathcal{G})$ is the union of triangles glued to each other along their vertices so that the spine is the trivalent tree illustrated in figure 1.

\subsection{Related complexes}

One can build a similar complex $X^{\prime}(\mathcal{G})$ with the vertex set $\mathcal{G}=\mathcal{A} / \Delta^{2}$. Each vertex has a special representative whose normal form either has no $\Delta$ 's, or has 


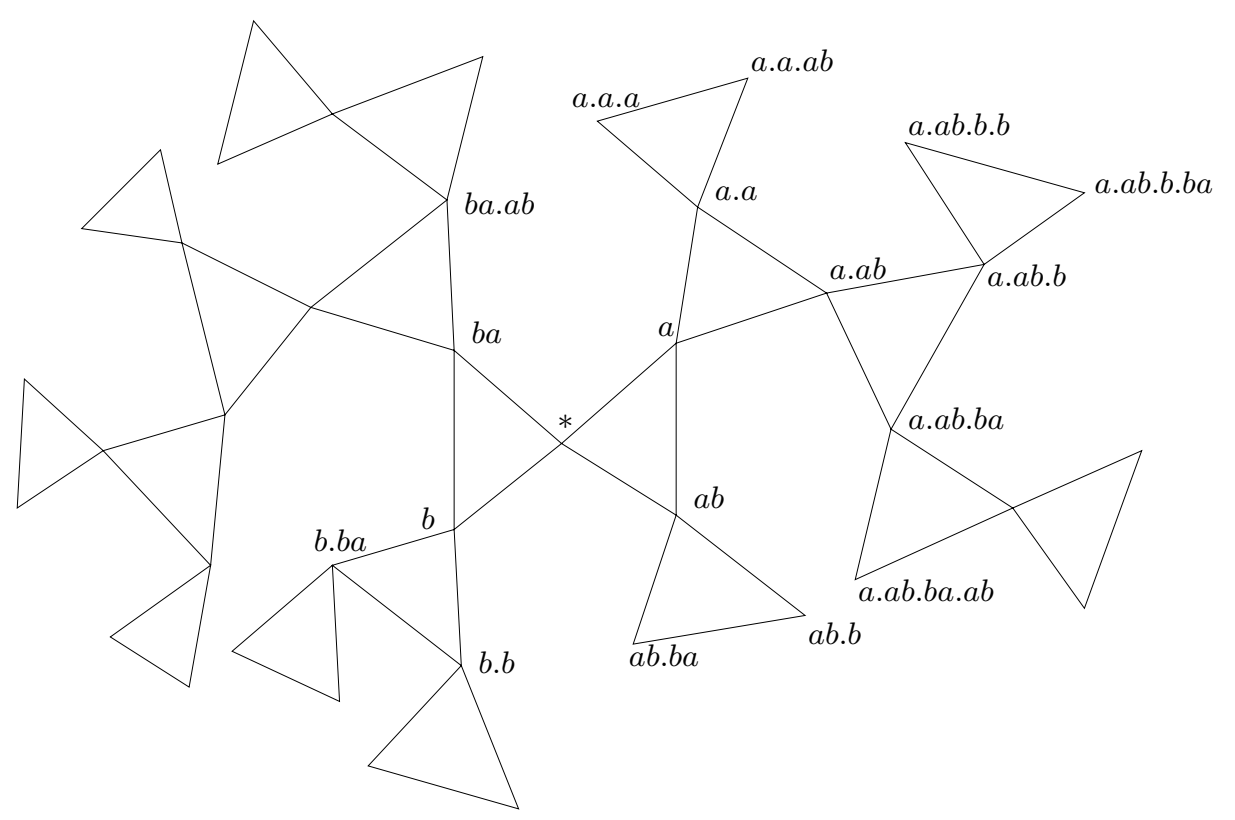

Figure 1: $X(\mathcal{G})$ for $\mathcal{G}=\mathcal{A} / \Delta^{2}, \mathcal{A}=\langle a, b \mid a b a=b a b\rangle$

a single $\Delta$, and in the latter case we agree to push this $\Delta$ to the last slot. There is an oriented edge from a vertex $v$ to a vertex $w$ if the special representative of $w$ is obtained from the special representative of $v$ by rightmultiplying by an atom. The simplicial complex $X^{\prime}(\mathcal{G})$ is defined to be the flag complex determined by the resulting graph, ie, a collection of vertices spans a simplex provided all pairs span an edge. The natural quotient map $\mathcal{G} \rightarrow \mathcal{G} /\langle\Delta\rangle$ extends to a simplicial map $p: X^{\prime}(\mathcal{G}) \rightarrow X(\mathcal{G})$. The preimage of the basepoint $* \in$ $X(\mathcal{G})$ is the edge $[*, \Delta] \subset X^{\prime}(\mathcal{G})$. More generally, the preimage of a simplex $\sigma$ is the (triangulated) prism $\sigma \times[0,1]$. There is a global homeomorphism $X^{\prime}(\mathcal{G}) \cong X(\mathcal{G}) \times[0,1]$. In the first coordinate this homeomorphism is given by $p$ and in the second it is the simplicial map that sends the vertices whose special representative has no $\Delta$ 's to 0 and the vertices whose special representative has one $\Delta$ to 1 .

Analogously, there is a complex $X(\mathcal{A})$ with vertex set $\mathcal{A}$ and similarly defined simplicial structure: edges are drawn from $*$ to the atoms and extended equivariantly, and then the higher-dimensional simplices are filled in. The quotient map extends to the natural simplicial map $q: X(\mathcal{A}) \rightarrow X(\mathcal{G})$ and there is a global homeomorphism $X(\mathcal{A}) \cong X(\mathcal{G}) \times \mathbb{R}$ in the first coordinate given by $q$ and in the second it is the map that is linear on each simplex and sends a vertex 
$g \in \mathcal{A}$ to length $(g) \in \mathbb{Z}$ (recall that length: $\mathcal{A} \rightarrow \mathbb{Z}$ is the homomorphism that sends each $s \in S$ to $1 \in \mathbb{Z})$. The fact that $\Delta^{2}$ virtually splits is transparent in this model.

We end this discussion by recalling (see [10]) the description of another important $\mathcal{A}$-complex and sketching the proof of its contractibility using the contractibility of $X(\mathcal{A})$ (Theorem 3.6).

The Coxeter sphere $\Sigma_{\mathcal{W}}$ can be described as (the geometric realization of) the poset of cosets $w \mathcal{W}_{F}$ where $w \in \mathcal{W}$ and $\mathcal{W}_{F}$ is the special Coxeter subgroup of $\mathcal{W}$ generated by $F \subset S$, as $F$ runs over all proper subsets (including $\emptyset$ ) of $S$. The partial order is given by inclusion. Here $\mathcal{W}_{F}$ corresponds to the barycenter of the largest face of $Q$ fixed by $F$ and $w \mathcal{W}_{F}$ corresponds to the $w$-translate of this face. One description [10] of the universal cover of the Salvetti complex $\tilde{\Sigma}_{\mathcal{W}}$ associated to $\mathcal{A}$ (and $\mathcal{W}$ ) is that it is the poset of subsets $a \mathcal{W}_{F} \subset \mathcal{A}$ with $a \in \mathcal{A}$ and $F$ any subset of $S$ (including $\emptyset$ and $S$ ), where $\mathcal{W} \supset \mathcal{W}_{F}$ is identified with the set of all atoms in $\mathcal{A}$. The Salvetti complex is the quotient of $\tilde{\Sigma}_{\mathcal{W}}$ by the pure Artin group. The Salvetti complex is homotopy equivalent to the associated hyperplane complement (see [10]).

The cover of $\tilde{\Sigma}_{\mathcal{W}}$ by the translates of $\mathcal{W}_{S}$ has the property that all nonempty intersections are contractible (this follows from the existence of least upper bounds for sets of atoms). Therefore, $\tilde{\Sigma}_{\mathcal{W}}$ is homotopy equivalent to the nerve of this cover.

Now consider the cover of $X(\mathcal{A})$ by the translates of the subcomplex spanned by all atoms. This cover also has all nonempty intersections contractible and its nerve is isomorphic to the nerve above. Thus $\tilde{\Sigma}_{\mathcal{W}}$ and $X(\mathcal{A})$ are homotopy equivalent.

\section{Geometric properties of $X(\mathcal{G})$}

\subsection{Geodesics}

There is a canonical "combing" of $X$ : for each vertex $v \in X$ we have a canonical edge-path from the basepoint $*$ to $v$. If $v=B_{1} \cdot B_{2} \cdots B_{k}$ is the normal form without $\Delta$ 's, then the edge-path associated to $v$ is

$$
*, B_{1}, B_{1} \cdot B_{2}, \cdots, B_{1} B_{2} \cdots B_{k}=v .
$$

Note that left multiplication by $\Delta$ sends $v$ to $\bar{B}_{1} \cdot \bar{B}_{2} \cdots \bar{B}_{k}$ and it sends the combing path from $*$ to $v$ to the combing path from $*=\Delta(*)$ to $\Delta(v)$. It 
follows that by left translating we obtain a canonical path from any vertex to any other vertex. We call all such edge-paths geodesics.

It is interesting that the geodesics are symmetric. This fact follows from the work of R Charney (Lemma 2.3 of [13]). For completeness we indicate a proof.

Proposition 3.1 The geodesic from $v$ to $w$ is the inverse of the geodesic from $w$ to $v$.

Proof First note that an edge-path is a geodesic if and only if every subpath of length 2 is a geodesic. Thus it suffices to prove the Proposition in the case that the geodesic from $v$ to $w$ has length 2. Further, after left-translating, we may assume that the middle vertex of the path is $*$, and so $v$ and $w$ are atoms, say $A$ and $B$ respectively. Denote by $C$ the atom with $C A=\Delta$. Note that $C$ is represented by a geodesic gallery from $-A$ to $Q$. We now have (recall our convention that we identify an atom $A$ with the chamber $\pi(A)(Q))$ :

$A, *, B$ is a geodesic $\Longleftrightarrow C A=\Delta=*, C, C B$ is a geodesic $\Longleftrightarrow C \cdot B$ is a normal form $\Longleftrightarrow$ (by Proposition 1.3, see figure 2) no wall $W=F i x(s)$ that abuts $Q$ has $Q$ and $-A$ on one side and $B$ on the other $\Longleftrightarrow$ (applying "-") no wall that abuts $-Q$ has $-Q$ and $A$ on one side and $-B$ on the other $\Longleftrightarrow$ (using that a wall abuts $Q$ iff it abuts $-Q$ and it always separates the two) no wall that abuts $Q$ has $Q$ and $-B$ on one side and $A$ on the other.

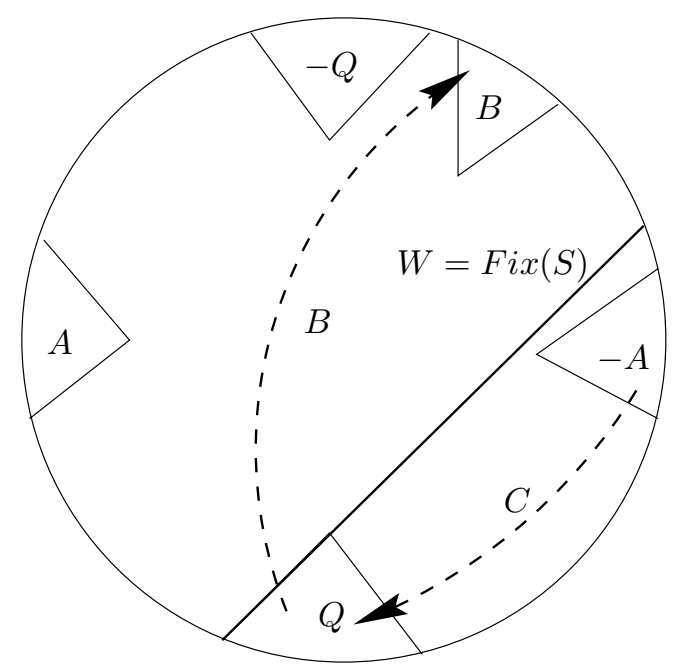

Figure 2: If $W$ exists, $C \cdot B$ is not a normal form; $C$ can be extended to a geodesic gallery $C^{\prime}=C s$ with $C^{\prime}<C B$. 
But the last statement is exactly the same as a previous statement with the roles of $A$ and $B$ interchanged.

Recall that if $A$ is an atom, the atom $A^{*}$ is defined by $A A^{*}=\Delta$. The following fact is contained in [13] (cf Lemmas 2.3 and 2.5).

Corollary 3.2 If $A_{1} \cdot A_{2} \cdots A_{k}$ is a normal form, so are $A_{k}^{*} \cdot \bar{A}_{k-1}^{*} \cdot A_{k-2}^{*} \cdots$.

Proof Left-translate the path $Q, A_{k}^{*}, A_{k}^{*} \bar{A}_{k-1}^{*}, \cdots$ by $A_{1} A_{2} \cdots A_{k}$ to get the path $A_{1} A_{2} \cdots A_{k}, A_{1} A_{2} \cdots A_{k-1}, \cdots, A_{1}, Q$. Since the inverse of this path is a geodesic by assumption, so is the original path by Proposition 3.1.

\section{$3.2 \quad$ Worddistance}

By the wordnorm of a vertex $v \in V$, denoted $\|v\|$, we mean the word-length of the special representative of $v$, or equivalently, the word-length of the normal form of any representative of $v$ after discarding the $\Delta$ 's. Observe that left translation by $\Delta$ is wordnorm preserving (it sends $B_{1} \cdot B_{2} \cdots B_{m}$ to $\bar{B}_{1}$. $\left.\bar{B}_{2} \cdots \bar{B}_{m}\right)$, and so there is a unique function $d_{w d}: V \times V \rightarrow \mathbb{Z}_{+}$that is leftinvariant and $d_{w d}(*, v)=\|v\|$ for all $v \in V$. We call $d_{w d}$ the worddistance, but we caution the reader that $d_{w d}$ is not symmetric. For example, if $B$ is an atom, then $d_{w d}(*, B)$ is the word-length of $B$, while $d_{w d}(B, *)$ is the word-length of the atom $C$ complementary to $B$ (ie such that $C B=\Delta$ ). This observation generalizes as follows.

Lemma 3.3 $d_{w d}(v, w)+d_{w d}(w, v)=d_{a t}(v, w) \delta$, where $\delta$ is the word-length of $\Delta$.

Proof After left-translating, we may assume that $v=*$. If $w=B_{1} \cdot B_{2} \cdots \cdots B_{k}$ (without $\Delta$ 's), then $d_{a t}(*, w)=k$ and $d_{w d}(*, w)$ is the word-length of $B_{1} \cdots B_{k}$. To compute $d_{w d}(w, *)$, denote by $C_{i}$ the atom with $C_{i} B_{i}=\Delta$. Left translate by $\cdots C_{3} \bar{C}_{2} C_{1}$ to get $d_{w d}(w, *)=d_{w d}\left(\Delta^{k}, \cdots C_{3} \bar{C}_{2} C_{1}\right)=\left\|\cdots C_{3} \bar{C}_{2} C_{1}\right\|$. Since the word-length of $C_{i}$ is obtained from $\delta$ by subtracting the word-length of $B_{i}$, the word-length of $\cdots C_{3} \bar{C}_{2} C_{1}$ equals $k \delta-d_{w d}(*, w)$, so it remains to argue that $\cdots C_{3} \cdot \bar{C}_{2} \cdot C_{1}$ is a normal form. But this is an immediate consequence of Corollary $3.2\left(C_{i}=\bar{B}_{i}^{*}\right)$. 
The triangle inequality $d_{w d}(v, w)+d_{w d}(w, z) \geq d_{w d}(v, z)$ is immediate, and so are the inequalities $d_{a t}(v, w) \leq d_{w d}(v, w) \leq \delta d_{a t}(v, w), d_{w d}(w, v) \leq \delta d_{w d}(v, w)$ (showing that $d_{w d}$ is "quasi-symmetric", and that $d_{w d}$ and $d_{a t}$ are quasiisometric).

We will next argue that the wordnorms of adjacent vertices are always distinct. To this end, we examine the relationship between the normal forms associated to two adjacent vertices, say $v=B_{1} \cdot B_{2} \cdots B_{k}$ and $w=C_{1} \cdot C_{2} \cdots \cdot C_{l}$ (without $\Delta$ 's). Since $d_{a t}(v, w)=1$, there is an atom $X \neq 1, \Delta$ such that $v X$ and $w$ represent the same coset, ie

$$
v X=w \Delta^{m}
$$

for some integer $m$. Similarly, there is a atom $Y \neq 1, \Delta$ such that

$$
w Y=v \Delta^{n}
$$

for an integer $n$.

Lemma 3.4 One of $m, n$ is 0 and the other is 1 .

Proof Substituting $v=w Y \Delta^{-n}$ into $v X=w \Delta^{m}$ yields $Y \Delta^{-n} X=\Delta^{m}$. If $n$ is even, this equation implies that $Y X$ is a power of $\Delta$, hence $Y X=\Delta$ and $m+n=1$. If $n$ is odd, then similarly $\bar{Y} X=\Delta$ and again $m+n=1$.

It remains to argue that $m, n \geq 0$. Assume $n<0$. Then $v=w Y \Delta^{-n}$ will have $\Delta$ 's in its normal form (as $\Delta$ can be moved to the front), contradiction. Similarly $m \geq 0$.

It now follows that either $\|v\|<\|w\|$ (if $m=0$ ) or $\|w\|<\|v\|$ (if $n=0$ ). We orient the edge $[v, w]$ from $v$ to $w$ if $\|v\|<\|w\|$. Thus the arrows point away from the basepoint. Note that these orientations are not group invariant.

It is clear from the definition that there are no oriented cycles. This implies that the dimension of $X(\mathcal{G})$ is length $(\Delta)-1$. Indeed, the set of vertices of a simplex of $X(\mathcal{G})$ is totally ordered and thus a top-dimensional simplex that, say, contains the base point $*$ corresponds to a chain of atoms $*<B_{1}<B_{2}<$ $\cdots<B_{m}$ of maximal possible length length $(\Delta)$.

The following lemma sharpens Lemma 2.1.

Lemma 3.5 Suppose $g=X_{1} X_{2} \cdots X_{k} \in \mathcal{A}^{+}$is written as the product of $k$ atoms, and let $g=B_{1} \cdot B_{2} \cdots B_{l}$ be the normal form for $g$. Then $l \leq k$ and $X_{1} X_{2} \cdots X_{i}<B_{1} B_{2} \cdots B_{i}$ for all $1 \leq i \leq l$. 
Proof That $l \leq k$ is the content of Lemma 2.1. That $X_{1}<B_{1}$ follows from the definition of the normal form. Assume inductively that $X_{1} \cdots X_{i-1}<$ $B_{1} \cdots B_{i-1}$. Thus we can write $B_{1} \cdots B_{i-1}=X_{1} \cdots X_{i-1} y$ for some $y \in \mathcal{A}^{+}$, and thus $X_{i} X_{i+1} \cdots X_{k}=y B_{i} B_{i+1} \cdots B_{l}$. But then

$$
X_{i}<\alpha\left(y B_{i} B_{i+1} \cdots B_{l}\right)=\alpha\left(y B_{i}\right)<y B_{i}
$$

so that multiplication on the left by $X_{1} \cdots X_{i-1}$ yields $X_{1} \cdots X_{i}<B_{1} \cdots B_{i}$. The equality in the displayed formula follows from Proposition 1.2.

\subsection{Contractibility of $X(\mathcal{G})$ and its topology at infinity}

Denote by $d=\operatorname{card}(S)-1$ the dimension of the Coxeter sphere (the unit sphere in $V_{\mathcal{W}}$ ).

In this section we prove:

Theorem 3.6 $X(\mathcal{G})$ is contractible and it is proper homotopy equivalent to a cell complex which is $d$-dimensional and $(d-2)$-connected at infinity.

In particular, this recovers a theorem of $\mathrm{C} C$ Squier [18]: $\mathcal{G}$ is a virtual duality group of dimension $d$ and $\mathcal{A}$ is a duality group of dimension $d+1$.

The method of proof is to consider the function $F: X \rightarrow[0, \infty)$ that to a vertex $v$ assigns the wordnorm $F(v)=\|v\|$ and is linear on each simplex. The theorem follows by standard methods (see [1], [2]) from:

Proposition 3.7 $F$ is nonconstant on each edge of $X(\mathcal{G})$. The ascending link at each vertex $v$ is either contractible or homotopy equivalent to the sphere $S^{d-1}$ and the descending link at each vertex $v \neq *$ is contractible.

For a vertex $v$, the ascending link $L k_{\uparrow}(v)$ [descending link $L k_{\downarrow}(v)$ ] is defined to be the link of $v$ in the subcomplex of $X(\mathcal{G})$ spanned by the vertices $w$ with $F(w) \geq F(v)[F(w) \leq F(v)]$.

We need a lemma. The proof was suggested by the referee and it is considerably simpler than the original argument.

Lemma 3.8 Given an atom $A$ and an element $b \in \mathcal{A}^{+}$there is a unique atom $C$ such that for every $u \in \mathcal{A}^{+}$

$$
A<b u \Longleftrightarrow C<u \text {. }
$$


Proof Let $d=A \vee b$. Then $d=b c$ for some $c \in \mathcal{A}^{+}$and

$$
A<b u \Leftrightarrow d<b u \Leftrightarrow c<u .
$$

Taking $u=\Delta$, we see that $c$ is an atom.

Proof of Proposition 3.7 Represent $v$ as a normal form $B_{1} \cdot B_{2} \cdots B_{k}$ without $\Delta$ 's. A representative of an adjacent vertex can be obtained from $B_{1} \cdots B_{k}$ by multiplying on the right by an atom, say $B$. Note that $F(v B)<$ $F(v)$ precisely when $\Delta<B_{1} \cdots B_{k} B$. By Lemma 3.8, there is an atom $C$ such that for any atom $B$

$$
\Delta<B_{1} \cdots B_{k} B \Longleftrightarrow C<B .
$$

Thus the descending link at $v$ can be identified with the poset of atoms $B \neq 1, \Delta$ such that $C<B$ and the ascending link with the poset of atoms $B \neq 1, \Delta$ such that $C \nless B$. The former is a cone with cone-point $C$ (unless $C=\Delta$ which corresponds to $v=*)$. To understand the latter, for each atom $D$ consider the subset $\mathcal{S}(D)=\cup\{B \mid B<D\}$ of the Coxeter sphere, where we now identify an atom $B$ with the intersection of the chamber $\pi(B)(Q)$ with the unit sphere in $V_{\mathcal{W}}$. The complement of $1 \cup \cup\{B \mid C<B\}$ is covered by the intersections with the sets $\mathcal{S}(D)$ for $C \nless D$. Proposition 1.1 implies that the collection of $\mathcal{S}(D)$ 's is closed under nonempty intersections. The elements are contractible (this can be seen by observing that for $C \nless D$ the convex ball $\mathcal{S}(D)$ intersects $\cup\{B \mid C<B\}$ in its boundary (if at all), it contains the simplex 1, and the boundary of $\mathcal{S}(D)$ intersects 1 in a proper collection of faces, ie, a ball, whose complement in $\partial(1)$ is also a ball), and thus the poset of the cover (which can be identified with the ascending link) is homotopy equivalent to the underlying space $S^{d} \backslash(1 \cup \cup\{B \mid C<B\})$. If the convex balls 1 and $\cup\{B \mid C<B\}$ are disjoint, then this complement is homotopy equivalent to $S^{d-1}$ and if they intersect then the complement is contractible.

Remark 3.9 The proof also shows that the dualizing module $H_{c}^{d}(X(\mathcal{G}) ; \mathbb{Z})$ is a free abelian group. In this situation, $H_{c}^{d}(X(\mathcal{G}) ; \mathbb{Z})$ is isomorphic to

$$
\bigoplus_{v \in V} H^{d-1}\left(L k_{\uparrow}(v, X(\mathcal{G})) ; \mathbb{Z}\right) .
$$

For details see [2].

Remark 3.10 There is another proof of the contractibility of $X(\mathcal{G})$ that follows the lines of Charney's Fellow Traveller Property [12]. One shows that the function $\phi: V \rightarrow V$ defined by $\phi(*)=*$ and

$$
\phi\left(A_{1} \cdot A_{2} \cdots A_{k-1} \cdot A_{k}\right)=A_{1} \cdot A_{2} \cdots A_{k-1}
$$


extends to a simplicial map $X(\mathcal{G}) \rightarrow X(\mathcal{G})$ which is homotopic to the identity. Then one observes that the image of any finite subcomplex of $X(\mathcal{G})$ under a high iterate of $\phi$ is a point.

Remark 3.11 The connectivity at infinity of right-angled Artin groups was studied by N. Brady and Meier [4]. These groups act cocompactly on CAT(0) spaces and the distance from a point is a good Morse function in that context. Brown and Meier [5] analyze which Artin groups of infinite type are duality groups.

\section{$3.4 \quad \mathrm{NPC}$}

We wish to study the properties of the distance function on $X(\mathcal{G})$, and in particular its "non-positively curved" aspects. We start by studying triangles formed by geodesics. It is convenient to translate the triangle so that one vertex is $*$. Denote the other two vertices by $v$ and $w$ (see figure 3 ).

Proposition 3.12 All edges in the geodesic from $v$ to $w$ whose orientation points towards $v$ occur at the beginning of the path, and these are followed by the edges oriented towards $w$.

In other words, the wordnorm first decreases and then increases along the geodesic.

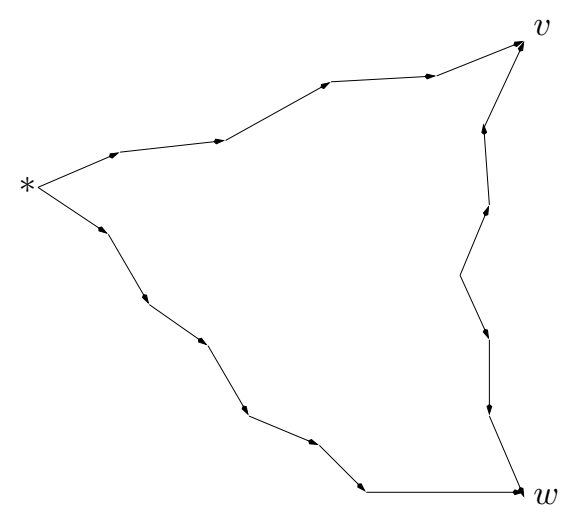

Figure 3: A geodesic triangle in $X(\mathcal{G})$

Proof Suppose on the contrary that $x, y, z$ are (special representatives of) consecutive vertices on the geodesic from $v$ to $w$ and that $\|x\|<\|y\|>\|z\|$. 
Thus there are atoms $B$ and $C$ such that $x B=y$ and $y C=z \Delta$. It follows that the first atom in the normal form for $x(B C)=z \Delta=\Delta \bar{z}$ is $\Delta$. Since $B \cdot C$ is a normal form, Proposition 1.2 implies that the first atom in the normal form for $x B=y$ is $\Delta$, a contradiction.

The following is the key "non-positively curved" feature of the complex $X(\mathcal{G})$. There are two versions, one for each metric. The $d_{w d}$-version is more useful, since the inequality is strict. It will allow us to run Cartan's fixed-point argument.

Theorem 3.13 Suppose $p$ is a vertex on the geodesic from $v$ to $w$ and $v \neq$ $p \neq w$. Let $u$ be any vertex. Then

$$
d_{a t}(u, p) \leq \max \left\{d_{a t}(u, v), d_{a t}(u, w)\right.
$$

and

$$
d_{w d}(u, p)<\max \left\{d_{w d}(u, v), d_{w d}(u, w)\right\}
$$

Moreover,

$$
d_{w d}(u, p) \leq \max \left\{d_{w d}(u, v), d_{w d}(u, w)\right\}-\min \left\{d_{a t}(v, p), d_{a t}(w, p)\right\} .
$$

Proof Without loss of generality $u=*$ and then the proof follows from Proposition 3.12 .

We remark that the function $d_{w d}(*, \cdot)$ is not necessarily convex along $[v, w]$, cf $\mathcal{A}=\mathbb{Z} \times \mathbb{Z} \times \mathbb{Z}$.

\subsection{Centers}

Let $T$ be a finite set of vertices of $X(\mathcal{G})$. The circumscribed radius $r(T)$ is the smallest integer $r$ such that for some vertex $v \in X, r=\max _{t \in T} d_{w d}(t, v)$. (Note the order of points $t$ and $v$ here.) A vertex $v$ is said to be a center of $T$ if $r(T)=\max _{t \in T} d_{w d}(t, v)$. A set might have more than one center, but we have the next best thing.

Proposition 3.14 If $v$ and $w$ are two centers of $T$, then $v$ and $w$ span an edge. 
Proof Suppose the geodesic from $v$ to $w$ passes through another vertex $z$. If $t \in T$, then

$$
d_{w d}(t, z)<\max \left\{d_{w d}(t, v), d_{w d}(t, w)\right\} \leq r(T)
$$

contradicting the definition of $r(T)$.

We next have the Cartan fixed point theorem in our setting.

Theorem 3.15 Every finite subgroup $H<\mathcal{G}$ leaves a simplex of $X(\mathcal{G})$ invariant (and fixes its barycenter). In particular, there are only finitely many conjugacy classes of finite subgroups in $\mathcal{G}$.

Proof Let $T$ be an orbit of $H$. The set of centers of $T$ is $H$-invariant and spans a simplex in $X(\mathcal{G})$.

\subsection{Minsets of elements}

In the theory of isometric actions on nonpositively curved spaces an important role is played by the minsets of isometries. These are the sets of points that move the least under the given isometry. Here we explore the analogous concept using the worddistance.

Proposition 3.16 Let $g \in \mathcal{G}$ and suppose $v, w$ are two vertices of $X(\mathcal{G})$ and $d_{w d}(v, g(v)) \geq d_{w d}(w, g(w))$. Then for each vertex $z$ on the geodesic from $v$ to $w$ we have $d_{w d}(z, g(z)) \leq d_{w d}(v, g(v))$.

Proof We may assume that $v=*$, by left-translating (and replacing $g$ by a conjugate). Let $w=X_{1} \cdot X_{2} \cdots X_{k}$ be the normal form without $\Delta$ 's. The element $g$ has the form $g=B_{1} \cdot B_{2} \cdots B_{m}$ (no $\Delta$ 's) or $g=B_{1} \cdot B_{2} \cdots B_{m} \Delta$.

Case $1 g=B_{1} \cdot B_{2} \cdots B_{m}$.

Note that the vertex $g(w)$ can be written as $B_{1} \cdots B_{m} X_{1} \cdots X_{k}$, and also as $X_{1} \cdots X_{k} u$ where $\|u\|=d_{w d}(w, g(w))$. Since we are assuming that $\|u\| \leq$ $\|g\|=d_{w d}(*, g(*))$, it follows that we have equality in $\mathcal{A}^{+}$:

$$
B_{1} \cdots B_{m} X_{1} \cdots X_{k}=X_{1} \cdots X_{k} u \Delta^{r}
$$

for some $r \geq 0$. In particular, $X_{1} \cdots X_{i}<B_{1} \cdots B_{m} X_{1} \cdots X_{i}$ (by Proposition 1.4) and so we have

$$
B_{1} \cdots B_{m} X_{1} \cdots X_{i}=X_{1} \cdots X_{i} u_{i}
$$


for some $u_{i} \in A^{+}$. In particular,

$$
d_{w d}\left(X_{1} \cdots X_{i}, g\left(X_{1} \cdots X_{i}\right)\right) \leq\left\|u_{i}\right\|=\left\|B_{1} \cdots B_{m}\right\|=\|g\|=d_{w d}(*, g(*)) .
$$

Case $2 g=B_{1} \cdot B_{2} \cdots B_{m} \Delta$.

The calculation is similar. We now get

$$
B_{1} \cdots B_{m} \Delta X_{1} \cdots X_{k}=X_{1} \cdots X_{k} u \Delta^{r}
$$

and $r \geq 1$ since $\|u\| \leq\|g\|=\left\|B_{1} \cdots B_{m}\right\|$. Now push the lone $\Delta$ on the lefthand side all the way to the right and cancel it to get $B_{1} \cdots B_{m} \bar{X}_{1} \cdots \bar{X}_{k}=$ $X_{1} \cdots X_{k} u \Delta^{r-1}$. Again by Proposition 1.4 we get

$$
X_{1} \cdots X_{i}<B_{1} \cdots B_{m} \bar{X}_{1} \cdots \bar{X}_{i}
$$

so that

$$
B_{1} \cdots B_{m} \bar{X}_{1} \cdots \bar{X}_{i}=X_{1} \cdots X_{i} u_{i}
$$

for some $u_{i} \in \mathcal{A}^{+}$with $\left\|u_{i}\right\|=\left\|B_{1} \cdots B_{m}\right\|=\|g\|$. Passing again to $\mathcal{A} /\langle\Delta\rangle$ we obtain $g\left(X_{1} \cdots X_{i}\right)=X_{1} \cdots X_{i} u_{i}$ and so

$$
d_{w d}\left(X_{1} \cdots X_{i}, g\left(X_{1} \cdots X_{i}\right)\right) \leq\left\|u_{i}\right\|=\|g\| .
$$

If $g \in \mathcal{G}$ and $d \in \mathbb{Z}$, let $T_{d}(g)=\left\{v \in X^{(0)} \mid d_{w d}(v, g(v)) \leq d\right\}$. Recall that $d_{w d}$ is not symmetric and note that writing $d_{w d}(g(v), v)$ instead in the definition would give $T_{d}\left(g^{-1}\right)$. The minimal $d$ with $T_{d}(g) \neq \emptyset$ is the displacement length of $g$, denoted $\lambda(g)$. The set $T_{d}(g)$ for this $d$ is the minset of $g$, denoted $A_{g}$.

A set $T$ of vertices is starlike with respect to $t_{0} \in T$ if for every $t \in T$ all vertices on the geodesic from $t_{0}$ to $t$ are in $T$. It can be shown, by the argument in Remark 3.10, that the span of a starlike set is contractible. A set of vertices is convex if it is starlike with respect to each of its vertices.

Corollary 3.17 The minset $A_{g}$ of each $g \in \mathcal{G}$ is convex.

Lemma 3.18 Let $g \in \mathcal{A}^{+}$have normal form $B_{1} \cdot B_{2} \cdots B_{k}$. Also suppose that the displacement of $*$ is not smaller than the displacement of the vertex $v$ with special representative $D_{1} \cdot D_{2} \cdots D_{l}$, ie, that $d_{w d}(*, g(*)) \geq d_{w d}(v, g(v))$. Then $D_{1} D_{2} \cdots D_{l}<B_{1} B_{2} \cdots B_{k} D_{1} D_{2} \cdots D_{l}$.

Proof Let $\tilde{D}=D_{1} D_{2} \cdots D_{l} . \quad d_{w d}\left(D_{1} \cdots D_{l}, g D_{1} \cdots D_{l}\right)$ is computed as follows: let $\tilde{E}=E_{1} \cdot E_{2} \cdots E_{l}$ be the normal form with $\tilde{E} \tilde{D}=\Delta^{l}$, find the normal form of $\tilde{E}\left(B_{1} \cdots B_{k} \tilde{D}\right)$, discard $\Delta$ 's and count letters. Since $d_{w d}(*, g(*)) \geq$ $d_{w d}(v, g(v))$, at least $l \Delta$ 's will have to be discarded, ie $\Delta^{l}<\tilde{E} B_{1} B_{2} \cdots B_{k} \tilde{D}$. Write $\Delta^{l}=\tilde{E} \tilde{D}$ and cancel $\tilde{E}$ to obtain $\tilde{D}<B_{1} B_{2} \cdots B_{k} \tilde{D}$. 


\section{Applications}

We now turn to the group-theoretic consequences of the discussion of the geometric properties of $X(\mathcal{G})$.

\subsection{Translation lengths}

The translation length of $g \in \mathcal{G}$, denoted $\tau(g)$, is defined to be

$$
\tau(g)=\lim _{n \rightarrow \infty} \frac{\left\|g^{n}(*)\right\|}{n} .
$$

See [17] for basic properties of translation functions (in the more general setting of finitely generated groups). An important feature of $\tau$ is that conjugate elements have the same translation length and the restriction of $\tau$ to a torsionfree abelian subgroup is a (non-symmetric) semi-norm. In [17], Gersten and Short prove that in biautomatic groups elements of infinite order have nonzero translation lengths, and this gives rise to many properties of such groups (eg a biautomatic group cannot contain the Heisenberg group as a subgroup). Finite type Artin groups are biautomatic by [12]. Here we show that translation lengths of torsion-free elements in $\mathcal{G}$ are bounded away from 0 , and this gives rise to additional group-theoretic properties.

Let $g \in \mathcal{G}$ be an element which is not conjugate to an atom, or an atom followed by $\Delta$ (we shall se momentarily that this forces $g$ to have infinite order). By $T_{n}$ denote the set $\left\{*, g(*), g^{2}(*), \cdots, g^{n-1}(*)\right\}$. Let $r_{n}$ be the circumscribed radius of $T_{n}$. Since $T_{1} \subset T_{2} \subset \cdots$ we have $r_{1} \leq r_{2} \leq \cdots$. Suppose that $r_{n}=r_{n+1}$ for some $n$. Let $z$ be a center for $T_{n+1}$. Then the ball of radius $r_{n}=r_{n+1}$ centered at $z$ covers both $T_{n}$ and $g\left(T_{n}\right)$ and so $z$ is a center for both. But then $g(z)$ is also a center for $g\left(T_{n}\right)$ and we conclude that $z$ and $g(z)$ span an edge, so that $g$ is conjugate to an atom or an atom followed by $\Delta$. Thus $r_{n} \geq n$ for all $n$ (and in particular $g$ has infinite order).

We now see that $\max _{i \leq n} d_{w d}\left(*, g^{i}(*)\right) \geq n$, and in particular there are infinitely many $n$ such that $d_{w d}\left(*, g^{n}(*)\right) \geq n$. It follows that the translation length $\tau(g) \geq 1$.

Recall that $\delta=\operatorname{length}(\Delta)$.

Theorem 4.1 The set of translation lengths of elements of $\mathcal{G}$ of infinite order is bounded below by $\frac{1}{2 \delta}$. 
Proof We have seen that if $g$ is of infinite order and not conjugate to $B$ or $B \Delta$ for an atom $B$, then $\tau(g) \geq 1$. Thus if $g^{2 \delta}$ is not conjugate to an atom or its inverse, then $\tau(g)=\frac{1}{2 \delta} \tau\left(g^{2 \delta}\right) \geq \frac{1}{2 \delta}$.

The length homomorphism length: $\mathcal{A} \rightarrow \mathbb{Z}$ descends to the homomorphism length: $\mathcal{G} \rightarrow \mathbb{Z} / 2 \delta$. Since conjugate elements have the same length, length $\left(g^{2 \delta}\right)$ $=0 \in \mathbb{Z} / 2 \delta$, and the only atom with 0 length is the identity element, it follows that if $g^{2 \delta}$ is conjugate to an atom or its inverse, then $g$ has finite order.

Corollary 4.2 Every abelian subgroup $A$ of $\mathcal{G}($ or $\mathcal{A}$ ) is finitely generated.

Proof Since $\mathcal{G}$ is virtually torsion-free, after passing to a finite index subgroup of $A$ we may assume that $A$ is torsion-free. Since the virtual cohomological dimension of $\mathcal{G}$ is finite, it follows that $A$ is a subgroup of the finite-dimensional vector space $W=A \otimes \mathbb{Q}$. Since $\tau: A \rightarrow[0, \infty)$ extends to a (non-symmetric) norm on $W$ and its values on $A \backslash\{0\}$ are bounded away from 0 , it follows that $A$ is discrete in $W$. (Strictly speaking, this is not a norm since $\tau$ is not symmetric. We could symmetrize, or else work with a non-symmetric norm which is just as good in the present context.)

Corollary 4.3 $\mathcal{G}$ does not have any infinitely divisible elements of infinite order.

The following corollary is a consequence of Theorem 3.5 of G. Conner's work on translation lengths [14]. For completeness, we sketch the proof.

Corollary 4.4 Every solvable subgroup of $\mathcal{G}($ or $\mathcal{A}$ ) is virtually abelian.

Proof Let $H$ be a solvable subgroup of $\mathcal{G}$. Since $\mathcal{G}$ is virtually torsion-free, we may assume that $H$ is torsion-free after replacing it with a subgroup of finite index. By induction on the length of the derived series, the commutator subgroup $[H, H]$ is virtually abelian, and hence finitely generated. Let $K \subset[H, H]$ be an abelian subgroup of finite index which is characteristic in $[H, H]$. The conjugation action of $H$ on $K$ is virtually trivial, since it preserves translation lengths (the image of the homomorphism $H \rightarrow G L(K)$ consists of elements of finite order and is therefore finite). Thus the action of $H$ on $[H, H]$ is virtually trivial as well. After passing to a subgroup of $H$ of finite index, we may assume that this action is trivial. The proof concludes by an argument in [17]. We now claim that $[H, H]$ is the trivial group, and so (the new) $H$ is abelian. Indeed, suppose that $x, y \in H$ and $z=[x, y]=x y x^{-1} y^{-1} \neq 1$. By our construction, $z$ commutes with both $x$ and $y$, and it has infinite order. We compute that $\left[x^{n}, y^{n}\right]=z^{n^{2}}$ and so the translation length of $z$ is 0 , a contradiction. 


\subsection{Finite subgroups of $\mathcal{G}$}

It is well-known to the experts that all finite subgroups of $\mathcal{G}$ are cyclic, and in fact the kernel of the homomorphism $\mathcal{G} \rightarrow \mathbb{Z} / 2 \delta$ is torsion-free, where $\delta=\|\Delta\|$ and the homomorphism is the length modulo $2 \delta$. (Note that the argument of Theorem 4.1 gives another proof of this fact.)

In this section we will use the geometric structure of $X(\mathcal{G})$ to give a classification of finite subgroups of $\mathcal{G}$ up to conjugacy.

Theorem 4.5 Every finite subgroup $H<\mathcal{G}$ is cyclic. Moreover, after conjugation, $H$ transitively permutes the vertices of a simplex $\sigma \subset X(\mathcal{G})$ that contains $*$ and $H$ has one of the following two forms:

Type 1 The order of $H$ is even, say $2 m$. It is generated by an atom $B$. The vertices of $\sigma$ are $*, B, B^{2}, \cdots, B^{m-1}$ (all atoms) and $B^{m}=\Delta$. Necessarily, $\bar{B}=B$ (since $\Delta$ fixes the whole simplex).

Type 2 The order $m$ of $H$ is odd, the group is generated by $B \Delta$ for an atom $B$, and the vertices $*, B, B \bar{B}, B \bar{B} B, \cdots,(B \bar{B})^{(m-1) / 2} B$ (all atoms) are permuted cyclically and faithfully by the group (so the dimension of $\sigma$ is $m-1$ ). Since $m$ is odd, the square $B \bar{B}$ of the generator also generates $H$.

An example of a type 1 group is $\langle B\rangle$ for $B=\sigma_{1} \sigma_{3} \sigma_{2}$ in the braid group $B_{4} /\left\langle\Delta^{2}\right\rangle$ (of order 4). An example of a type 2 group is $\left\langle\sigma_{1} \Delta\right\rangle$ in $B_{3} /\left\langle\Delta^{2}\right\rangle$ (of order 3 ).

The key to this is:

Lemma 4.6 The set of vertices of any simplex $\sigma$ in $X$ admits a cyclic order that is preserved by the stabilizer $\operatorname{Stab}(\sigma)<\mathcal{G}$.

Proof We can translate $\sigma$ so that $*$ is one of its vertices. Let the cyclic order be induced from the linear order $*<B_{1}<B_{2}<\cdots<B_{k}$ given by the orientations of the edges of $\sigma$ (equivalently, by the lengths of the atoms $\left.B_{i}\right)$. We need to argue that the left translation by $B_{i}^{-1}$ is going to produce the same cyclic order. We can write $B_{i+1}=B_{i} C_{i}$ for atoms $C_{i}$, so that the vertices of $\sigma$ are $*, C_{1}, C_{1} C_{2}, \cdots, C_{1} \cdots C_{k}$. After translation by $B_{1}^{-1}$ the vertices are $B_{1}^{-1}, *, C_{2}, C_{2} C_{3}, \cdots, C_{2} \cdots C_{k}$, where $B_{1}^{-1}$ should be replaced by the special representative of the coset $B_{1}^{-1}\langle\Delta\rangle$. Let $Y$ be the atom with $C_{1} C_{2} \cdots C_{k} Y=\Delta$. Then $B_{1}^{-1} \Delta=C_{1}^{-1} \Delta=C_{2} C_{3} \cdots C_{k} Y$ and $C_{2} C_{3} \cdots C_{k} Y$ is this canonical representative (it is a subword of $\Delta$, so it is an atom). Since $C_{2} \cdots C_{k}<C_{2} \cdots C_{k} Y$, it follows that the induced ordering on the vertices is a cyclic permutation of the old one. Repeating this $i$ times gives the claim. 
Proof of Theorem 4.5 Recall that by Theorem 3.15 there is an $H$-invariant simplex $\sigma \subset X(\mathcal{G})$. By passing to a face if necessary and conjugating, we may assume that $H$ acts transitively on the vertices of $\sigma$, and that $*$ is a vertex of $\sigma$. Say there are $m$ vertices and choose $h \in H$ that rotates the simplex by one unit. Note that $h^{m}$ is either 1 or $\Delta$. Also, $h$ is either an atom $B$ or else $h=B \Delta$ for an atom $B$. In the first case, the vertices of the simplex are $*, B, B B, \cdots, B^{m-1}$ and these are all atoms (since the arrow points from $B^{i}$ to $B^{i+1}$ for each $\left.i=0,1, \cdots, m-2\right)$. Thus $1<B^{m-1}<\Delta$ and hence $B<B^{m}<B \Delta$, so it follows that $B^{m}=\Delta$, and $H$ is of type 1. In the second case, the vertices of $\sigma$ are $*, B, B \bar{B}, B \bar{B} B, \cdots$ and they are similarly all atoms. If $m$ is even, the last vertex in this sequence is $(B \bar{B})^{m / 2-1} B$, and this is still an atom. It follows that $(B \bar{B})^{m / 2}=\Delta$. But then $h^{m}=\Delta \in H$ fixes the whole simplex and in particular $B=\bar{B}$ and $H$ is of type 1 . So suppose $m$ is odd. Then the last vertex of $\sigma$ is $(B \bar{B})^{\frac{m-1}{2}}$ and we have $(B \bar{B})^{\frac{m-1}{2}} B=\Delta$. Multiplication by $\Delta$ on the right reveals that $h^{m}=1$ and $H$ is of type 2 .

\subsection{Normal abelian subgroups and centers of finite index sub- groups}

We use minsets to prove:

Theorem 4.7 Assume that the associated Coxeter group $\mathcal{W}$ is nonabelian and irreducibe. The action of $\mathcal{G}$ on itself by conjugation does not have nontrivial finite orbits. (Singletons consisting of central elements are trivial orbits.) In particular, the center of any finite index subgroup of $\mathcal{G}$ is either trivial or $\langle\Delta\rangle$ (if the latter is central in $\mathcal{G}$ ).

We remark that $\Delta$ is central in $\mathcal{G}$ if and only if it is central in $\mathcal{A}$ (if $\Delta g=g \Delta$ in $\mathcal{G}$ then a priori we only get $\Delta g=g \Delta^{2 m+1}$ in $\mathcal{A}$, but $m$ must be 0 by length considerations) if and only if the bar involution is trivial.

We now turn to the lemmas needed in the proof.

Lemma 4.8 Suppose $A_{1}, \cdots, A_{p}$ is a finite collection of convex sets in $V=$ $\mathcal{G} /\langle\Delta\rangle$ permuted by left translations. Then each $A_{i}$ is empty or all of $V$.

Proof If not, we may assume $\emptyset \neq A_{i} \neq V$ for each $i$, by discarding the copies of $\emptyset$ and $V$ from the collection. Let $q \geq 1$ be the largest integer such that for some $i_{1}<i_{2}<\cdots<i_{q}$ the intersection

$$
\bigcap_{j=1}^{q} A_{i_{j}} \neq \emptyset .
$$


Now pass to the collection of $q$-fold intersections of the $A_{i}$ 's. We can therefore assume that the sets in the collection, still denoted $A_{i}$, are pairwise disjoint. Since $\mathcal{G}$ acts transitively on $V$, the sets $A_{i}$ must cover $V$. Say $* \in A_{1}$. Let $\sigma$ be a generator of $\mathcal{A}^{+}$and consider the line $L=\left\{\sigma^{j}(*) \mid j \in \mathbb{Z}\right\}$. By convexity, if $\sigma^{j}(*), \sigma^{l}(*) \in A_{i}$, then $\sigma^{k}(*) \in A_{i}$ for $k$ between $j$ and $l$ (this is because the normal form of $\sigma \sigma \cdots \sigma$ is $\sigma \cdot \sigma \cdots \cdots \sigma)$. We conclude that for $j$ large each $\sigma^{j}(*)$ belongs to the same $A_{i}$. Left translation by $\sigma^{-j}$ takes $\sigma^{j}(*) \in A_{i}$ to $* \in A_{1}$ and it takes $\sigma^{2 j}(*) \in A_{i}$ to $\sigma^{j}(*) \in A_{i}$, and so $i=1$. Convexity now implies that $\sigma^{j}(*) \in A_{1}$ for all $j \geq 0$, and in particular $\sigma(*) \in A_{1}$.

Let $v$ be now any vertex and assume $v \in A_{1}$. Choose $g \in \mathcal{G}$ with $g(v)=*$. Thus $g\left(A_{1}\right)=A_{1}$ and we then have $g^{-1}(\sigma(*)) \in A_{1}$. The two choices of $g$ give $v \sigma \in A_{1}$ and $v \bar{\sigma} \in A_{1}$. Since any vertex can be reached from $*$ by successively right-multiplying by a generator, we conclude $A_{1}=V$, a contradiction.

It is convenient to introduce the following notion. Recall that $x_{1} \cdot x_{2} \cdots x_{k}$ is a normal form if and only if $x_{i}=\alpha\left(x_{i} x_{i+1}\right)$ for all $i$. Motivated by this observation we construct, following $\mathrm{R}$ Charney [12], a finite graph whose vertex set is the set of atoms not equal to 1 or $\Delta$, and there is an arrow from $x$ to $y$ if $x=\alpha(x y)$. A nontrivial normal form without $\Delta$ 's is simply a finite directed path in this graph. We refer to this graph as the Charney graph. So the elements of $V$ are in 1-1 correspondence with oriented paths in the Charney graph (with $*$ corresponding to the empty path, and atoms corresponding to one point paths).

Proposition 4.9 If $\mathcal{W}$ is irreducible, then any two atoms $x, y$ not equal to 1 or $\Delta$ can be joined by an oriented path in the Charney graph.

Proof As the first case, we assume that $x$ and $y$ are generators. Let $x=$ $g_{1}, g_{2}, \cdots g_{k}=y$ be a sequence of generators such that successive elements do not commute (this sequence exists by the irreducibility assumption). Then

$$
g_{1} \cdot g_{1} g_{2} \cdot g_{2} \cdot g_{2} g_{3} \cdot g_{3} \cdots g_{k-1} \cdot g_{k-1} g_{k} \cdot g_{k}
$$

is the desired path.

Next, we observe that if $x \neq 1$ is any atom, then there is a generator $g$ such that $x \cdot g$ is a normal form. Simply take $g$ to be the last generator in a word representing $x$.

It remains to argue that if $y$ is any atom not equal to 1 or $\Delta$, there is a generator $g$ and an oriented path from $g$ to $y$. Let $S$ be the set of generators $a$ such 
that some word representing $y$ begins with $a$. If $S$ consists of a single element $a$, then $a \cdot g$ is a normal form and we are done. We proceed by induction on the cardinality of $S$. We first note that $S$ is a proper subset of the generating set (if a chamber is separated from the fundamental chamber $Q$ by every wall adjacent to $Q$, then the chamber is the antipodal chamber $-Q$ ). Next, observe that $\Delta(S) \cdot y$ is a normal form, where $\Delta(S)$ is the $\Delta$-element in the subgroup generated by $S$. Let $c$ be a generator not in $S$. Then $c \Delta(S)$ is an atom that can begin with $c$ or with an element of $S$ that commutes with $c$ (this follows from the cancellation law and the fact that if $y$ begins with $c$ and $x$ then it begins with $\operatorname{prod}(c, x, m(c, x)))$. Since $c \Delta(S) \cdot \Delta(S) \cdot y$ is a normal form, we have replaced the original set $S$ by the set $S^{\prime}$ consisting of $c$ and the elements of $S$ that commute with $c$.

Case $1 c$ can be chosen so that at least two elements of $S$ don't commute with $c$.

Then $\operatorname{card}\left(S^{\prime}\right)<\operatorname{card}(S)$ and we are done by induction.

Recall that the Coxeter graph is a tree, and consider the forest spanned by $S$. Two generators commute if and only if they are not adjacent in the Coxeter graph.

Case 2 The forest has more than one component.

If there are two components separated by a single vertex $c$, then clearly $\operatorname{card}\left(S^{\prime}\right)$ $<\operatorname{card}(S)$ and we are done. Otherwise choose $c$ to be adjacent to one component while separating it from another, and so that the distance between the two components is as small as possible. Then $S^{\prime}$ has the same cardinality as $S$, but the associated forest has two components that are closer together than in the old forest. Repeating this procedure eventually produces two components separated by a single vertex.

Case 3 The forest is a tree and $\operatorname{card}(S)>1$.

Then choose $c$ to be adjacent to a vertex in the tree. $S^{\prime}$ has the same cardinality as $S$ but the underlying forest has $>1$ component.

Lemma 4.10 Suppose $\mathcal{W}$ is irreducible. Then for any chamber $R$ and any wall $W$ that abuts $R$ there is a normal form $D_{1} \cdot D_{2} \cdots D_{l}$ such that

- $D_{l}$ is a single generator,

- The gallery associated to $D_{1} D_{2} \cdots D_{l}$ that starts at the fundamental chamber $Q$ ends at the chamber $R$ and last crosses wall $W$. 
Moreover, if $B \neq 1, \Delta$ is a given atom, the normal form can be chosen so that $B \cdot D_{1}$ is a normal form.

Proof Using the connectivity of the Charney graph, start with a normal form $E_{1} \cdot E_{2} \cdots E_{m}$ so that $E_{m}$ is a single generator (and $B \cdot E_{1}$ is a normal form). Say $R^{\prime}$ is the terminal chamber of the gallery $E_{1} \cdots E_{l}$ and the last wall crossed is $W^{\prime}$. If $X$ is a generator that does not commute with $E_{m}$, then $E_{1} \cdot E_{2} \cdots E_{m} \cdot E_{m} \cdot\left(E_{m} X\right) \cdot X$ is also a normal form whose gallery ends at $R^{\prime}$, but the last wall crossed is a different wall from $W^{\prime}$. By irreducibility, any two generators can be connected by a sequence of generators with successive generators noncommuting, and thus we can construct a gallery as above that ends at $R^{\prime}$ and last crosses any preassigned wall abutting $R^{\prime}$. By repeating the last atom in such a normal form, we can construct a similar gallery that ends in any preassigned chamber adjacent to $R^{\prime}$ and by iterating these operations we can get to $R$ and $W$.

Lemma 4.11 If $\mathcal{W}$ is irreducible, then $A_{g} \neq V$ for every nontrivial $g \in \mathcal{G}$, unless $g=\Delta$ and $\Delta$ is central.

We will actually prove a stronger form of this lemma. Consider the set $H \subset \mathcal{G}$ of elements $g \in \mathcal{G}$ such that the function $v \mapsto d_{w d}(v, g(v))$ defined on $V$ is bounded above. It is then clear that $H$ is a normal subgroup of $\mathcal{G}$.

Proposition 4.12 If $\mathcal{W}$ is irreducible, then $H$ is either trivial or equal to $\langle\Delta\rangle$ (if $\Delta$ is central).

Proof We will first argue that every element of $H$ has finite order. This will imply that $H$ is finite (since $H \rightarrow \mathcal{W}$ will then be injective).

Let $g \in H$ have infinite order. Notice that $\left\|g^{i}(*)\right\| \rightarrow \infty$ as $i \rightarrow \infty$. This is because $g^{i}(*)=g^{j}(*)$ for $i \neq j$ would imply that $g^{i-j}$ equals 1 or $\Delta$, so $g$ would have finite order. Fix a large number $N$ (to be specified later) and replace $g$ by a power if necessary so that $\|g(*)\|>N$. Further, by conjugating $g$ if necessary, we may assume that $\|g(*)\|=d_{w d}(*, g(*))$ realizes the maximum of the (bounded) function $v \mapsto d_{w d}(v, g(v))$.

As usual, we have either $g=B_{1} \cdot B_{2} \cdots B_{k}$ or $g=B_{1} \cdot B_{2} \cdots B_{k} \Delta$. Note that $k>N / \delta$ is large.

Case $1 g=B_{1} \cdot B_{2} \cdots B_{k}$. 
If $\tilde{D}=D_{1} \cdot D_{2} \cdots D_{l}$ is a normal form such that $B_{k} \cdot D_{1}$ is a normal form, then consider the vertex $D_{1} \cdots D_{l}(*)=D_{1} \cdots D_{l}$. From Lemma 3.18 we get $D_{1} D_{2} \cdots D_{l}<B_{1} B_{2} \cdots B_{k} D_{1} D_{2} \cdots D_{l}$. Assume in addition that $l \leq$ $k$. Since the right-hand side is also a normal form, Lemma 2.1 implies that $D_{1} D_{2} \cdots D_{l}<B_{1} B_{2} \cdots B_{l}$. We will use this only when $l=k$. Summarizing, if $B_{k} \cdot D_{1}$ is a normal form, then $D_{1} \cdots D_{k}<B_{1} \cdots B_{k}$. But we now argue that we can choose $\tilde{D}=D_{1} \cdot D_{2} \cdots D_{k}$ so that $D_{1}$ follows $B_{k}$ (in the Charney graph) but $\tilde{D} \nless B_{1} \cdots B_{k}$.

Say $M$ is an integer such that the atomnorm of the galleries constructed in Lemma 4.10 is bounded by $M$.

Each atom $B \neq \Delta$ crosses at most $\delta-1$ walls in the Coxeter sphere. Thus $B_{1} \cdots B_{k}$ crosses at most $k(\delta-1)$ walls. Therefore some wall $W$ is crossed by $B_{1} \cdots B_{k}$ at most $k(\delta-1) / \delta$ times and the same is true for any initial piece of $B_{1} \cdots B_{k}$. We will get a contradiction (for large $N$ and $k$ ) by arguing that $\tilde{D}$ can be chosen so that it crosses a preassigned wall at least $k-M$ times (contradiction arising if $k-M>k(\delta-1) / \delta$ ie, if $k>\delta M$, and this can be arranged if $N>\delta^{2} M$ ).

Start with a normal form $D_{1} \cdot D_{2} \cdots D_{l}$ with $l \leq M$ so that $B_{k} \cdot D_{1}$ is a normal form, $D_{l}$ is a single generator and the last wall crossed is $W$. This is possible by Lemma 4.10 (a left translation may be necessary before applying the lemma since $B_{1} \cdots B_{k}$ may end at a chamber different from $Q$ ). Then set $D_{i}=D_{l}$ for $i=l+1, l+2, \cdots, k$.

Case $2 g=B_{1} \cdots B_{k} \Delta$.

This is entirely analogous, except for some overlines, and is left to the reader.

The proof of the proposition then follows from the following result.

Proposition 4.13 If $\mathcal{W}$ is irreducible and $H<\mathcal{G}$ is a finite normal subgroup, then $H$ is trivial or $H=\langle\Delta\rangle$ with $\Delta$ central.

Proof Since $H$ is finite, there is an $H$-invariant simplex in $X(\mathcal{G})$. Since $H$ is normal, every vertex belongs to an $H$-invariant simplex. If $H=\langle\Delta\rangle$, then $*$ is fixed by $H$, and hence every vertex is fixed by $H$. Thus the bar involution is trivial and $\Delta$ is central. If $H$ is nontrivial and not equal to $\langle\Delta\rangle$, then $H$ must contain a nontrivial atom $B \neq \Delta$ (the inverse of an element of the form $B \Delta$ is an atom). Left translation by $B$ moves every vertex to an adjacent vertex, and so $d_{w d}(v, B v)<\delta$ for every vertex $v$. On the other hand, for any $g \in \mathcal{G}$ the numbers $d_{w d}(v, g(v))$ are all congruent $\bmod \delta$ to each other, as $v$ ranges 
over the vertices of $X(\mathcal{G})$, so in our situation we see that all displacements $d_{w d}(v, B v)$ are equal to each other. By the irreducibility of $\mathcal{W}$, there is a normal form $B \cdot B_{1} \cdot B_{2} \cdots B_{k}$ with $B_{k}$ any prechosen atom. Lemmas 3.18 and 2.1 now imply that $B_{1} \cdots B_{k}<B B_{1} \cdots B_{k-1}$. In particular, we see that $\left\|B_{k}\right\| \leq\|B\|$. By choosing $B_{k}$ to have length $\delta-1$, we conclude that $B$ has length $\delta-1$. There is then a generator $\sigma$ such that $\sigma B=\Delta$ and we conclude that $B^{-1}=\Delta \sigma \in H$. But $\Delta \sigma$ has infinite order, unless $\sigma$ and $\bar{\sigma}$ are the only generators. (To see this, note that $X \cdot X \cdot X \cdots X$ is a normal form where $X=\operatorname{prod}(\sigma, \bar{\sigma}, m(\sigma, \bar{\sigma}))$.) If $\sigma=\bar{\sigma}$, then there is only one generator and $G=\langle\Delta\rangle \cong \mathbb{Z}_{2}$ so there is nothing to prove. If $\sigma \neq \bar{\sigma}$, then each half of the Artin relation $\sigma \bar{\sigma} \sigma \cdots=\bar{\sigma} \sigma \bar{\sigma} \cdots$ has length equal to an odd integer $m \geq 3$. In particular, $\sigma \bar{\sigma} \in H$ is an atom, so it must have length $\delta-1$, and this forces $m=3$. We are now reduced to the classical braid group on 3 strands modulo the center. To finish the argument, note that in this case $\sigma \bar{\sigma} \cdot \bar{\sigma}$ is a normal form, while $\bar{\sigma} \nless \sigma \bar{\sigma}$.

Proof of Theorem 4.7 Let $g_{1}, \cdots, g_{n}$ be a finite orbit under conjugation. Note that there is $K>0$ such that $d_{w d}\left(v, g_{i}(v)\right) \leq K$ for all $i=1,2, \cdots, n$ and all vertices $v$. Indeed, we can take $K=\max d_{w d}\left(*, g_{i}(*)\right)$, for then

$$
d_{w d}\left(v, g_{i}(v)\right)=d_{w d}\left(h(*), g_{i} h(*)\right)=d_{w d}\left(*, h^{-1} g_{i} h(*)\right)=d_{w d}\left(*, g_{j}(*)\right)
$$

where $h$ is chosen so that $h(*)=v$. It now follows from Proposition 4.12 that each $g_{i}$ is central.

The following corollary answers a question of Jim Carlson. It motivated the construction of $X(\mathcal{G})$ and the analysis of its geometric properties.

Corollary 4.14 Assume that the associated Coxeter group $\mathcal{W}$ is irreducible. Let $A$ be a normal abelian subgroup of $\mathcal{G}$. Then $A$ is trivial or $\langle\Delta\rangle$ (and in the latter case $\Delta$ is central).

Proof $\mathcal{G}$ acts on $A$ by conjugation. If $A$ is not as in the conclusion, then this action has infinite orbits by Theorem 4.7. The abelian group $A$ is finitely generated by Corollary 4.2. The translation length function induces a norm on the free abelian group $A$ /torsion. The induced action of $\mathcal{G}$ must preserve this norm and it still has infinite orbits, a contradiction. 


\section{The space at infinity}

We now construct a "space at infinity" of $X(\mathcal{G})$ and examine the basic properties of the action of $\mathcal{G}$. In a joint work with Mark Feighn it will be shown that elements of infinite order have periodic points at infinity. This is to be regarded as the analog of the space of projectivized geodesic measured laminations for the case of mapping class groups (when $\mathcal{A}$ is a braid group, $\mathcal{G}=\mathcal{A} / \Delta^{2}$ is a mapping class group).

\subsection{Definition}

Recall that a normal form without $\Delta$ 's is an oriented path in the Charney graph.

Definition 5.1 An admissible itinerary is an infinite directed path $X=x_{1}$. $x_{2} \cdot x_{3} \cdots$ in the Charney graph.

We denote by $\Omega$ the set of all admissible itineraries and topologize it in the usual fashion: two are close if they agree for a long time. Thus $\Omega$ is a totally disconnected compact metrizable space, and it is nonempty unless $\mathcal{A}$ is trivial or $\mathbb{Z}$. If $\mathcal{W}$ is irreducible and nonabelian, then $\Omega$ is a Cantor set, by Proposition 4.9 plus the observation that there are vertices with at least two outgoing edges (eg if $a, b \in S$ don't commute then there are oriented edges from $a$ to both $a$ and $a b)$. We now describe an action of $\mathcal{A}^{+}$on $\Omega$. Let $X \in \Omega$ be as above and let $g \in \mathcal{A}^{+}$. Observe that by Proposition $1.2 y_{1}=\alpha\left(g x_{1}\right)=\alpha\left(g x_{1} x_{2} \cdots x_{k}\right)$ for any $k \geq 1$. Similarly, by Proposition 1.4 the second atom $y_{2}$ in the normal form for $g x_{1} x_{2}$ equals the second atom in the normal form for $g x_{1} x_{2} \cdots x_{k}$ for any $k \geq 2$. Continuing in this fashion, we see that the normal forms for $g x_{1} x_{2} \cdots x_{k}$ "converge" as $k \rightarrow \infty$ to an infinite sequence $y_{1} \cdot y_{2} \cdot y_{3} \cdots$ so that any finite initial piece is a normal form. This may not be an admissible itinerary since $y_{i}$ might be $\Delta$. However, observe that only finitely many $y_{i}$ 's can be $\Delta$. They all occur at the beginning and their number is no larger than the atomnorm of $g$. If the number of $\Delta$ 's is even we erase them, and if the number is odd we erase them and replace all the remaining $y_{i}$ by their "conjugate" $\bar{y}_{i}$. Intuitively, we think of pushing all the $\Delta$ 's off to infinity, much in the same way as we calculate the special representative of a vertex of $X(\mathcal{G})$.

Since $\Delta^{2}$ acts trivially, there is an induced action of $\mathcal{G}$ on $\Omega$. The action is continuous (this follows from Lemma 1.4). 


\subsection{Faithfulness and minimality}

Proposition 5.2 If $\mathcal{A}$ is not $1, \mathbb{Z}$, or $\mathbb{Z} \times \mathbb{Z}$, then the kernel of the action of $\mathcal{G}=\mathcal{A} / \Delta^{2}$ on $\Omega$ is either trivial or $\langle\Delta\rangle$ (if $\Delta$ is central).

Proof If $\Delta$ is not central, then there is a generator $a$ such that $\bar{a}=b \neq a$. Then $a^{\infty}:=a \cdot a \cdot a \cdots \in \Omega$ is not fixed by $\Delta$; indeed its image is $b^{\infty}$.

Let $g \in \mathcal{A}^{+}$be nontrivial. Assume first that the normal form of $g=x_{1} \cdot x_{2} \cdots x_{k}$ does not have any $\Delta$ 's. Let $a$ be a generator such that $x_{k} \cdot a$ is a normal form (ie, so that $x_{k} a$ is not an atom - eg $a$ can be the last letter in a word representing $\left.x_{k}\right)$. Then $a^{\infty}:=a \cdot a \cdot a \cdots \in \Omega$ is fixed by $g$ only if $g=a^{k}$ is a power of $a$. If $b$ is a generator that does not commute with $a$, then $a^{k}\left(b^{\infty}\right)=a \cdot a \cdots a \cdot a b \cdot b \cdot b \cdot \cdots$ and $b^{\infty}$ is not fixed by $g$. Finally, if $b$ is a generator distinct from $a$ and commuting with $a$, but there are other generators (so that $\Delta \neq a b$ ), then $a^{k}\left(b^{\infty}\right)=a b \cdot a b \cdots a b \cdot b \cdot b \cdots$, so $b^{\infty}$ is not fixed in that case either.

Finally, assume that the normal form of $g$ is $\Delta \cdot x_{1} \cdots x_{k}$ with $k \geq 1$ and $x_{1} \neq \Delta$. Again take $a$ to be a generator with $x_{k} \cdot a$ a normal form. If $g$ fixes $a^{\infty}$, then $\bar{x}_{1} \cdots \bar{x}_{k} \cdot \bar{a} \cdot \bar{a} \cdots=a \cdot a \cdots$ and so $g=\Delta a^{k}$ and $\bar{a}=a$. If $b$ is a generator such that $\bar{b}$ does not commute with $a$, then $\Delta a^{k}\left(b^{\infty}\right)=$ $a^{k} \bar{b}^{\infty}=a \cdot a \cdots \cdots \cdot a \bar{b} \cdot \bar{b} \cdots$ and so $b^{\infty}$ is not fixed. Finally, if $\mathcal{A}$ is abelian but has more than 2 generators and if $b$ is a generator distinct from $a$, then $\Delta a^{k} b^{\infty}=a b \cdot a b \cdots \cdots a b \cdot b \cdot b \cdot \cdots$.

Proposition 5.3 Suppose that $\mathcal{W}$ is irreducible and nonabelian. Then each orbit in $\Omega$ is dense. In particular, there is no proper closed invariant subset of $\Omega$.

Proof Let $X=x_{1} \cdot x_{2} \cdots$ and $Y=y_{1} \cdot y_{2} \cdots$ be two points in $\Omega$. We need to construct some $g \in \mathcal{A}^{+}$such that $g X$ and $Y$ agree in the first $n$ slots. By Proposition 4.9 there is a finite directed path $y_{n} \cdot z_{1} \cdots z_{k} \cdot x_{1}$ from $y_{n}$ to $x_{1}$. Take $g=y_{1} y_{2} \cdots y_{n} z_{1} \cdots z_{k}$.

\section{References}

[1] M Bestvina, N Brady, Morse theory and finiteness properties of groups, Invent. Math. 109 (1997) 445-470

[2] M Bestvina, M Feighn, The topology at infinity of Out $\left(F_{n}\right)$, preprint (1997) 
[3] M Bridson, A Haefliger, Metric spaces of non-positive curvature, manuscript of a book, in progress

[4] N Brady, J Meier, Connectivity at infinity for right-angled Artin groups, preprint

[5] K S Brown, J Meier, Improper actions and higher connectivity at infinity, in progress

[6] N Bourbaki, Groupes et Algebres de Lie, Masson, Paris (1981) Chapters IV-VI

[7] T Brady, Artin groups of finite type with three generators, preprint

[8] E Brieskorn, Sur les groupes de tresses, Sémininaire Bourbaki 24e année, 1971/72, no. 401, LNM, vol. 317, Springer-Verlag (1973) 21-44

[9] E Brieskorn, K Saito, Artin Gruppen und Coxeter Gruppen, Invent. Math. 17 (1972) 245-271

[10] R Charney, M Davis, Finite $K(\pi, 1)$ s for Artin groups, from: "Prospects in Topology", Proceedings of a Conference in honor of William Browder, (F Quinn, editor), Annals of Math. Studies, vol. 138, Princeton University Press (1995) 110-124.

[11] R Charney, M Davis, The $K(\pi, 1)$-problem for hyperplane complements associated to infinite reflection groups, Jour. Amer. Math. Soc. 8 (1995) 597-627

[12] R Charney, Artin groups of finite type are biautomatic, Math. Ann. 292 (1992) 671-683

[13] R Charney, Geodesic automation and growth functions for Artin groups of finite type, Math. Ann. 301 (1995) 307-324

[14] G Conner, Discreteness properties of translation numbers in solvable groups, preprint

[15] P Deligne, Les immeubles des groupes de tresses généralisés, Invent. Math. 17 (1972) 273-302

[16] F A Garside, The braid groups and other groups, Quart. J. Math. Oxford 20 (1969) 235-254

[17] S Gersten, H Short, Rational subgroups of biautomatic groups, Ann. Math. 105 (1991) 641-662

[18] C C Squier, The homological algebra of Artin groups, Math. Scand. 75 (1994) 5-43 